\title{
Strongly Enhanced Low Energy $\alpha$-Particle Decay in Heavy Actinide Nuclei and Long-Lived Superdeformed and Hyperdeformed Isomeric States
}

\author{
A. Marinov ${ }^{(1)}$, S. Gelberg( ${ }^{(1)}$, D. Kolb ${ }^{(2)}$ and J. L. Weil ${ }^{(3)}$ \\ (1) Racah Institute of Physics, The Hebrew University, Jerusalem 91904, Israel \\ (2) Department of Physics, Kassel University, 34109 Kassel, Germany \\ (3) Department of Physics and Astronomy, University of Kentucky, Lexington, KY 40506, USA
}

\begin{abstract}
Relatively low energy and very enhanced $\alpha$-particle groups have been observed in various actinide fractions produced via secondary reactions in a CERN W target which had been irradiated with $24-\mathrm{GeV}$ protons. In particular, 5.14, 5.27 and $5.53 \mathrm{MeV} \alpha$-particle groups with corresponding half-lives of $3.8 \pm 1.0 \mathrm{y}, 625 \pm 84 \mathrm{~d}$ and $26 \pm 7 \mathrm{~d}$, have been seen in Bk, Es and Lr-No sources, respectively. The measured energies are a few MeV lower than the known ground state to ground state $\alpha$-decays in the corresponding neutrondeficient actinide nuclei. The half-lives are $10^{4}$ to $10^{7}$ shorter than expected from the systematics of $\alpha$-particle decay in this region of nuclei. The deduced evaporation residue cross sections are in the mb region, about $10^{4}$ times higher than expected. A consistent interpretation of the data is given in terms of production of long-lived isomeric states in the second and third wells of the potential-energy surfaces of the parent nuclei, which decay to the corresponding wells in the daughters. The possibility that the isomeric states in the third minimum are actually the true or very near the true ground states of the nuclei, and consequences regarding the production of the long-lived superheavy elements, are discussed.
\end{abstract}

PACS numbers: 23.60.+e, 21.10.Tg, 25.60.Pj, 27.90.+b

\section{INTRODUCTION}

In a study of the radioactive decay of various actinide fractions [1,2] separated from a CERN W target which had been irradiated with $24-\mathrm{GeV}$ protons [3 5], very unusual phenomena which could not be understood from nuclear systematics, had been observed.

First [2], isomeric states with $\mathrm{t}_{1 / 2} \sim 0.6 \mathrm{y}$ and $\geq 30 \mathrm{~d}\left(10^{4}-10^{5}\right.$ times longer than the expected half-lives of the corresponding ground states) were found in neutron-deficient

${ }^{236} \mathrm{Am}$ and ${ }^{236} \mathrm{Bk}$ nuclei, respectively. About $3 \times 10^{5}$ atoms of ${ }^{236} \mathrm{Am}$ and $4 \times 10^{4}$ atoms of ${ }^{236} \mathrm{Bk}$ were produced in the isomeric states, and decayed by the $\beta^{+}$or electron capture processes. The character of these states was not clear: they are far from closed shells where high spin isomers are usually found, and they have very long lifetimes as compared to the known shape isomers. 
Secondly, long-lived fission activities with half-lives of several years [四, have been seen in all the actinide sources from Am up to Fm, and perhaps also in the Md-No and the No-Lr sources. Also here the origin of such long-lived fission activities is not known.

Another observation was very low energy, 3.0 and $4.0 \mathrm{MeV}$, particle groups, which were seen in the Am source in coincidence with $\mathrm{L}_{\alpha 1} \mathrm{X}$-rays in the Am region [1]. The estimated lifetimes [6] deviate by about 23 and 12 orders of magnitude respectively from those obtained from the systematic relationship between $\alpha$-particle energies and their lifetimes [7]. It therefore was assumed [6] that the observed particle groups were protons of unknown origin rather than $\alpha$-particles.

Perhaps the most surprising phenomenon was the relatively low energy and very enhanced $\alpha$-particle groups, observed in various actinide sources which were separated from the $\mathrm{W}$ target [1, 2,6]. For instance, a $5.14 \mathrm{MeV}$ group with a half-life of about $4 \mathrm{y}$ has been seen in the Bk source, and a $5.53 \mathrm{MeV}$ group with a half-life of about $26 \mathrm{~d}$, has been seen in the Lr-No source. One is faced here with the following problems:

a) The long lifetimes of the nuclei as compared to corresponding ground state systematics: It is expected and in fact evident from the identification of the ${ }^{236} \mathrm{Bk}$ and ${ }^{236} \mathrm{Am}$ nuclei, that neutron-deficient actinide nuclei can be produced via the secondary reactions in the $\mathrm{W}$ target. Lifetimes of several years for neutron-deficient nuclei around Bk, and of about a month in the Lr-No region, are very much longer than the expected minutes or hours in the first case, and seconds in the second case. Here also one is led to the conclusion that long-lived isomeric states of unknown character, which decay by the observed $\alpha$-activities, were produced in the reaction.

b) The problem of the low energy of the $\alpha$-particles: An energy of $5.14 \mathrm{MeV}$ in the neutron-deficient Bk region is a low energy as compared to typically 6-7 MeV, and 5.53 $\mathrm{MeV}$ is very low for Lr-No nuclei, where the typical transition energies are above $8 \mathrm{MeV}$. The penetrability factor for $6 \mathrm{MeV} \alpha$-particles in the Bk region is about 5 orders of magnitude larger than for $5.14 \mathrm{MeV}$, and for $8 \mathrm{MeV}$ in the No region is about 13 orders of magnitude larger than for $5.53 \mathrm{MeV}$ [7]. Naturally, one assumes that an $\alpha$-decay from an isomeric state is of even higher energy than normal, because of the larger available transition energy. One is therefore faced with the problem, what is causing the nucleus to decay with very low-energy $\alpha$-particles, when in principle much higher transition energy is available with correspondingly many orders of magnitude larger penetrability factor.

c) The problem of the very enhanced character [6] of the $\alpha$-decay: According to systematics [7], the half-lives for $5.14 \mathrm{MeV} \alpha$-particles for Bk and nearby nuclei, and for $5.53 \mathrm{MeV}$ $\alpha$-particles in Lr-No, are around $10^{5}$ to $10^{6} \mathrm{y}$. The question is how the $\alpha$-particle decay rate can be enhanced by 5 to 7 orders of magnitude.

d) The problem of the large cross section of the heavy-ion reaction: The production yield in the secondary reaction experiment depends on two cross sections: i) The cross section for production of suitable fragments with high enough kinetic energies, which may interact with other $\mathrm{W}$ nuclei in the target, and lead to the formation of the various actinide nuclei. ii) The heavy ion reaction, or the evaporation residue, cross section, which depends first on the fusion cross section between such a fragment and another W nucleus in the target, and secondly on the competition with the fission process during the cooling of the produced compound nucleus. Using reasonable estimates about the first process, evaporation residue cross sections in the region of $\mathrm{mb}$ are needed in order to explain the production of about $10^{4}$ 
actinide atoms seen experimentally. These are unexpectedly large cross sections, particularly when the production of nuclei in the Lr-No region is considered where the typical evaporation residue cross sections are below $1 \mu \mathrm{b}$ [8].

Recently 911 some similar abnormal decay phenomena have been observed, for the first time, in several ordinary heavy-ion reaction studies. In a study of the ${ }^{16} \mathrm{O}+{ }^{197} \mathrm{Au}$ reaction at $\mathrm{E}_{L a b}=80 \mathrm{MeV}$ [9], an isomeric state has been found in ${ }^{210} \mathrm{Fr}$ which decays by emitting a relatively low energy $\alpha$-particle group, with $\mathrm{E}_{\alpha}=5.20 \mathrm{MeV}$ and $\mathrm{t}_{1 / 2} \sim 90 \mathrm{~m}$. Since this half-life is longer than the known half-life of the ground state of ${ }^{210} \mathrm{Fr}\left(\mathrm{t}_{1 / 2}=3.18\right.$ $\mathrm{m}$ ), it was concluded that a long-lived isomeric state had been formed in this nucleus. A $\mathrm{t}_{1 / 2}$ of $90 \mathrm{~m}$ for $5.20 \mathrm{MeV} \alpha$-particles in ${ }^{210} \mathrm{Fr}$ is enhanced by a factor of $3 \times 10^{5}$ as compared to normal transitions [7,9]. However, this $\alpha$-group was observed in coincidence with $\gamma$-rays which fit predictions for a superdeformed band [9]. Therefore the effect of large deformations of the nucleus on the $\alpha$-particle decay was calculated and found [9] to be consistent with the observed enhancement. It was argued 90 that since the isomeric state decays to a high spin state, it should also have high spin, and since it decays by enhanced $\alpha$-particle emission to state(s) in the second well of the potential, it should be in the second well itself.

In the same reaction two long-lived proton activities with half-lives of about $6 \mathrm{~h}$ and 70 $\mathrm{h}$ were found [10] with proton energies of $1.5-4.8 \mathrm{MeV}$ with a sharp line at 2.19 MeV. A possible interpretation in terms of production of long-lived isomeric states in the second well of the potential of the parent nucleus which decay by protons to the normal states of the daughter was given [10].

Very recently [11], long-lived isomeric states, produced by the ${ }^{28} \mathrm{Si}+{ }^{181} \mathrm{Ta}$ reaction at bombarding energies of 125 and $135 \mathrm{MeV}$, which decay by strongly hindered $\alpha$-particle and proton activities, have been discovered. In particular, a very retarded $8.6 \mathrm{MeV} \alpha$-particle group with $40 \mathrm{~d} \leq \mathrm{t}_{1 / 2} \leq 2.1 \mathrm{y}$ (as compared to less than a $1 \mu \mathrm{s}$, typical for a normal transition of such high energy) was found in coincidence with $\gamma$-rays of a superdeformed band. This group has been consistently interpreted [1] in terms of production of a longlived isomeric state in the third (hyperdeformed) well probably of ${ }^{195} \mathrm{Hg}$, which decays by very retarded $\alpha$-particles, to the second (superdeformed) well in ${ }^{191} \mathrm{Pt}$.

In the present work it is shown that the low energy and very enhanced $\alpha$-particle groups, seen in the heavy actinide, Bk, Es and Lr-No sources, can be consistently interpreted as due to formation of long-lived isomeric states in the second and third wells of the potential energy surfaces in the parent nuclei, which decay to the corresponding wells in the daughters. Furthermore, it is pointed out that according to theoretical predictions [12], the third minima are the actual ground states of the very heavy actinide nuclei.

\section{EXPERIMENTAL PROCEDURE AND RESULTS}

The experimental procedure has been published before [1] and will be briefly reviewed. Actinide fractions from Am up to Lr-No were separated [1, 2] from our (W3) [3,4] tungsten

target (6 cm long, $30 \mathrm{~g}$, 99.95\% pure) which was irradiated in CERN with about $1 \times 10^{18}$ 
$( \pm 20 \%)$ protons of $24 \mathrm{GeV}$ energy. Fig. 1 shows a block diagram of the chemical procedure. The main aim in the chemical separation was to separate the heavy actinides, from Am up to Lr, from the rare-earth isotopes and from $\alpha$-emitting nuclei such as Po, Ra, Ac, Th, U and $\mathrm{Pu}$. Several anion (Dowex-1) and cation (Dowex-50) exchange columns were used for this purpose, and at the end two fractions were obtained. The first included the remainder of the rare-earth elements, Am and part of the $\mathrm{Cm}$. The second fraction included the other part of $\mathrm{Cm}$ and all the heavier actinides. These two fractions were then passed through Dowex-50 cation exchange columns with ammonium $\alpha$-hydroxyisobutyrate acid at $87^{\circ} \mathrm{C}$, in order to separate individual actinides. The elution curves obtained from these last separations are shown in fig. 2. The $\mathrm{X}$-rays of the rare-earth elements, measured with a $\mathrm{Si}(\mathrm{Li})$ detector were used throughout the whole procedure to identify the contents of the fractions. Actinide sources were prepared as indicated in fig. 2 using the measured elution positions of the rareearth elements, and known information on the actinides [13]. The sources were prepared on thin glass discs which were then heated to about $600^{\circ} \mathrm{C}$ to remove any organic material present.

The decontamination factor for $\mathrm{Th}$ and $\mathrm{Pu}$ was estimated to be $\geq 10^{8}$ [2]; the decontamination factor from a typical rare earth element like $\mathrm{Gd}$, determined by a comparison of the residue number of ${ }^{148} \mathrm{Gd}$ nuclei seen in the Bk and Cf sources [1] with the total number of produced ${ }^{148} \mathrm{Gd}$ nuclei, deduced from its known production cross section [14], was about $9 \times 10^{4}$. The intensity of ${ }^{148} \mathrm{Gd}$ was found to be about 4000 times larger in the $\mathrm{Cf}$ and $\mathrm{Bk}$ sources (where it should preferentially be) compared to the adjacent Es and Cm sources [1].

$\alpha$-particle spectra were measured from the various sources using $450 \mathrm{~mm}^{2} \mathrm{Si}$ surface barrier detectors. In the present paper we concentrate on the 5.14, 5.27 and $5.53 \mathrm{MeV}$ groups which have been seen with the Bk, Es and Lr-No sources, respectively. The uncertainty in the energies was about $\pm 40 \mathrm{keV}$. Fig. 3 (top) shows a typical $\alpha$-particle spectrum obtained with the Bk source. (Fig. If of ref. [2] represents a corresponding background spectrum). A pronounced peak at $5.14 \mathrm{MeV}$ is seen in this spectrum. (The other pronounced peak at $5.74 \mathrm{MeV}$ was identified to be due to the decay of ${ }^{236} \mathrm{Pu}$, obtained after several $\beta^{+}$or EC transitions from an isomeric state in ${ }^{236} \mathrm{Bk}[$ [国). Fig. 3 (bottom) gives the decay curve of the $5.14 \mathrm{MeV}$ group, measured during about $15 \mathrm{y}$. It is seen in this figure that the intensity first grows with a half-life of $2.0 \pm 0.5 \mathrm{y}$ and then decays with a half-life of $3.8 \pm 1.0 \mathrm{y}$. Fig. 4 shows similar plots obtained with the Es source, where a $5.27 \mathrm{MeV} \alpha$-particle group with a half-life of about $625 \mathrm{~d}$ is seen. In fig. 5 two spectra obtained with the Lr-No source are shown. In the first spectrum (top) a pronounced peak at $5.53 \mathrm{MeV}$ is seen, while in the second one (bottom), which was measured about 3 months later, very little of this peak remained. From these two spectra a half-life of $26 \pm 7 \mathrm{~d}$ is obtained. (Fig. 1e of ref. [2] is the corresponding background spectrum for fig. 4 (top) and fig. 5). From the measured intensities and lifetimes of the $\alpha$-particle groups, it was estimated that the number of produced atoms were $3.3 \times 10^{4}$, $1.4 \times 10^{4}$ and $1.4 \times 10^{4}$, in the Bk, Es and Lr-No sources, respectively. These number are correct to within $\pm 50 \%$.

\footnotetext{
${ }^{1}$ The chemical separation was performed by the late A. M. Friedman.
} 


\section{DISCUSSION}

It is impossible to identify the above mentioned $\alpha$-particle groups with any known activity in the whole nuclear chart. We shell now show in some detail that one can rule out the possibility that the $5.14 \mathrm{MeV}$ group is due to ${ }^{208} \mathrm{Po}\left(\mathrm{E}_{\alpha}=5.12 \mathrm{MeV} ; \mathrm{t}_{1 / 2}=2.898 \mathrm{y}\right)$, the $5.27 \mathrm{MeV}$ group is due to ${ }^{210} \mathrm{Po}\left(\mathrm{E}_{\alpha}=5.30 \mathrm{MeV} ; \mathrm{t}_{1 / 2}=138.4 \mathrm{~d}\right)$, and the $5.53 \mathrm{MeV}$ group is due ${ }^{222} \mathrm{Rn}\left(\mathrm{E}_{\alpha}=5.49 \mathrm{MeV} ; \mathrm{t}_{1 / 2}=3.83 \mathrm{~d}\right)$.

1) The $5.14 \mathrm{MeV}$ group in fig. 3 is not due to ${ }^{208}$ Po since:

a) In the chemical procedure (fig. 1) there were 4 anion exchange columns where Po should have been strongly adsorbed.

b) $P_{0}$ is very volatile. The last stage in the preparation of the sources, as mentioned above, was heating them to $600^{\circ} \mathrm{C}$. If some Po was left after the chemical separation, then it would have evaporated during this heating.

c) As mentioned above the half-life of ${ }^{208}$ Po is 2.9 years and it does not have a long-lived parent. If the $5.14 \mathrm{MeV}$ is from ${ }^{208} \mathrm{Po}$, then the intensity at the beginning in fig. 3 (bottom) should have been about twice as much as the intensity seen at around $3 \mathrm{y}$, namely $160 \pm 12$ and not $20 \pm 20$ as seen. In addition the intensity at the last point in fig. 3 (bottom) should have been $5 \pm 0.4$ and not $20 \pm 6$ as seen.

2) The $5.27 \mathrm{MeV}$ group seen with the Es source in fig. 4 is not due to ${ }^{210}$ Po since:

a) (1a) and (1b) above.

b) The deduced half-life is $625 \pm 85 \mathrm{~d}$ which is about 4.5 times longer than the known half-life of ${ }^{210} \mathrm{Po}$ of $138.4 \mathrm{~d}$.

This group is also not due to ${ }^{210} \mathrm{Po}$ which is fed from ${ }^{210} \mathrm{~Pb}$ because:

c) In the chemical separation procedure (fig. 1) there were one $1.5 \mathrm{M} \mathrm{HCl}$ anion exchange and three cation exchange columns where $\mathrm{Pb}$ should have been adsorbed.

d) The half-life of ${ }^{210} \mathrm{~Pb}$ is $22.3 \mathrm{y}$ and of ${ }^{210} \mathrm{Po}$ is $138.4 \mathrm{~d}$. By normalizing to the data around $120 \mathrm{~d}$ and using mother-daughter relationship for these half-lives one gets that the intensity in the first measurement shown in fig. 4 (bottom) should have been $23 \pm 3$ counts, and in the last measurement $290 \pm 32$. These figures are in complete contradiction to the observed data where the corresponding numbers are $135 \pm 22$ and $40 \pm 5$, respectively.

3) The $5.53 \mathrm{MeV}$ group seen with the Lr-No source in fig. 5 is not due to ${ }^{222} \mathrm{Rn}$ (which is introduced to the counters with air) for the following reasons:

a) If the $5.53 \mathrm{MeV}$ group is from ${ }^{222} \mathrm{Rn}$ then groups from its short-lived daughters ${ }^{218} \mathrm{Po}_{\mathrm{O}}$ and ${ }^{214} \mathrm{Po}$, of about the same intensity, should have been seen at $6.00 \mathrm{MeV}$ and $7.69 \mathrm{MeV}$ in fig. 5 (top). Nothing is seen in these energy regions.

b) A group from the air should have been very narrow, about $25 \mathrm{keV}$, and not about 130 $\mathrm{keV}$, as seen experimentally in fig. 5 .

c) One does not see such a group of similar intensity in other spectra which were measured under identical conditions like the one displayed in fig. 5 (bottom), or in the relevant background spectrum which is shown in fig. 1e of Ref. [2].

It is also not due to ${ }^{222} \mathrm{Rn}$ which is fed from ${ }^{226} \mathrm{Ra}$, since one does not see in fig. 5 (top) the $4.78 \mathrm{MeV} \alpha$-particle group of ${ }^{226} \mathrm{Ra}$, the half-life of ${ }^{226} \mathrm{Ra}$ is $1600 \mathrm{y}$ much longer than the measured half-life of $26 \pm 7 \mathrm{~d}$, and also because of (3a) above. 
$\alpha$-particle decays with similar energies but with very different half-lives of a few seconds are known in very neutron-deficient $\mathrm{Yb}$ to $\mathrm{Pt}$ nuclei. For instance, ${ }^{167} \mathrm{Re}$ and ${ }^{180} \mathrm{Pt}$ decay with $\alpha$-particles of about $5.14 \mathrm{MeV} ;{ }^{174 m} \mathrm{Ir},{ }^{174} \mathrm{Ir},{ }^{166} \mathrm{Re},{ }^{170} \mathrm{Os},{ }^{160 m} \mathrm{Ta},{ }^{158} \mathrm{Hf},{ }^{154} \mathrm{Yb}$ and ${ }^{160} \mathrm{Ta}$ decay with $\alpha$-particles around $5.3 \mathrm{MeV}$, and ${ }^{156} \mathrm{Lu}$ with a $5.57 \mathrm{MeV}$ group. In principle one may assume that the activities seen by us are due to such decays which are fed by unknown long-lived isomeric states. This possibility has been studied by us in some detail. Six of the above mentioned isotopes, those above $\mathrm{W}$, can in principle be produced by either $(\mathrm{p}, \mathrm{xn})$ or secondary reactions, where the number of evaporated neutrons is between 9 to 17. For instance, ${ }^{167}$ Re can be reached by the ${ }^{182} \mathrm{~W}(\mathrm{p}, 16 \mathrm{n}){ }^{167}$ Re reaction, and ${ }^{174} \mathrm{Ir}$ by ${ }^{182} \mathrm{~W}\left({ }^{6} \mathrm{Li}, 14 \mathrm{n}\right){ }^{174} \mathrm{Ir}$ reaction. Additionally, five of the above mentioned isotopes, those below $\mathrm{W}$, can in principle be produced by spallation reactions where $12-13$ neutrons have to be evaporated beyond the most neutron deficient isotopes which are known to be produced by such reactions. By using very modest assumptions about the production cross sections of these nuclei, [2 and very modest assumptions or measurements of the decontamination factors of our sources from these elements, ${ }^{5}$ it was estimated that the probabilities that all these isotopes, except ${ }^{154} \mathrm{Yb}$ and ${ }^{156} \mathrm{Lu}$, will be produced and present in our sources are extremely small, factor of $10^{5}$ to $10^{16}$ below the experimental values. (In addition, there are other arguments which prove that some of these isotopes are not those present in our spectra, such as the missing of competing $\alpha$-particle groups or characteristic $\alpha$-groups of the daughters decays. For instance, the $5.27 \mathrm{MeV}$ group seen in the Es source can not be due to ${ }^{174 m} \mathrm{Ir}$, since one does not see in fig. 4 its 7.3 times stronger $\alpha$-group at $5.48 \mathrm{MeV}$ ).

As far as ${ }^{154} \mathrm{Yb}$ and ${ }^{156} \mathrm{Lu}$ are concerned, the $5.27 \mathrm{MeV}$ group seen in the Es source is not due to ${ }^{154} \mathrm{Yb}$, for the following reasons: a) it is not seen in the Lr-No source, where $\mathrm{Yb}$ should be present with about 400 times stronger intensity, and b) one does not see in Fig. 4 the decay of its descendant, ${ }^{150} \mathrm{Dy}$, at $4.23 \mathrm{MeV}$ with a total estimated intensity of about

\footnotetext{
${ }^{2}$ For instance, for the production of ${ }^{167}$ Re from the ${ }^{182} \mathrm{~W}(\mathrm{p}, 16 \mathrm{n}){ }^{167}$ Re reaction, a cross section of $1.5 \mathrm{mb}$ was assumed for the production of ${ }^{178} \mathrm{Re}$, like in the production of ${ }^{177} \mathrm{~W}$ from ${ }^{181} \mathrm{Ta}$ [15], and this was reduced by a factor of 5 for each additional evaporated neutron (Fig. 3a in Ref. [16]). This factor is very modest. It may be much larger for very neutron-deficient nuclei where the proton binding energy decreases and the neutron binding energy increases. (The binding energy for a proton in ${ }^{167} \mathrm{Re}$ is about $260 \mathrm{keV}$ and for a neutron it is about $11 \mathrm{MeV}$ ). For the secondary reactions, the cross sections for the required high energy (above the corresponding Q-values) heavy ions, like $\alpha$-particles, ${ }^{6} \mathrm{Li}$ and ${ }^{7} \mathrm{Be}$, were estimated from Ref. [17], and evaporation residue cross sections of $1 \mu \mathrm{b}, 1 \mathrm{nb}$ and $1 \mathrm{pb}$ were assumed for $9 \mathrm{n}, 14 \mathrm{n}$, and $16 \mathrm{n}$ reactions, respectively. These are rather modest assumptions. For the spallation reactions, known cross sections [15, 18] for the production of the most neutron-deficient nucleus were used, and then reduced by a factor of 5 for each additional (12 or 13) evaporated neutron (see above).

${ }^{3}$ For Hf, Yb and Lu the decontamination factors were measured by a comparison with the X-ray intensities from a $0.4 \%$ of the solution of the $\mathrm{LaF}_{3}$ precipitate before the main chemical separation took place (see Fig. 1). For the other elements it was assumed that the decontamination factor due to adsorption in an anion or a cation exchange column is at least as large as the decontamination factor of $\mathrm{Yb}$ in the Es source which was determined to be about 1200 (see Fig. 2 (top)).
} 
11 counts. (Only 2 counts, which may also be background events, are seen in this energy region. The probability to see 2 events when 11 are expected is $\left.1 \times 10^{-3}\right)$. Likewise, the 5.53 $\mathrm{MeV}$ group seen in the Lr-No source is not due to ${ }^{156} \mathrm{Lu}$, since one does not see in Fig. 5 the decay of its descendant, ${ }^{152} \mathrm{Er}$, at $4.8 \mathrm{MeV}$, with $90 \%$ intensity as compared to the 5.53 $\mathrm{MeV}$ group.

Thus it is seen that one may rule out the possibility that the experimentally observed $\alpha$-particle groups are due to unknown long-lived isomeric states in the neutron-deficient $\mathrm{Yb}$ to Pt nuclei.

Another possibility may perhaps be that the observed $\alpha$-particle groups are due to unknown long-lived isomeric states in the rare-earth region. This possibility is very unlikely for the following reasons:

a) The decay properties of the rare-earth nuclei were studied very thoroughly, including measuring of extremely small branching ratios of $\alpha$-particles, using reactions which were particularly chosen for the production of each isotope by itself. There is no known long-lived isomeric state which decays by relatively high-energy $\alpha$-particles ( $5-5.5 \mathrm{MeV}$ as compared to around $3 \mathrm{MeV}$ ) in the whole rare-earth region. It is very difficult to see how such unknown activities will be produced and be present in our sources, which were separated from the rare-earth elements quite thoroughly (the decontamination factor of the Bk and Es sources from their rare-earth homologs was about $10^{5}$ (see section II above), and that of the Lr-No source from $\mathrm{Yb}$ and $\mathrm{Lu}$ was about $4.5 \times 10^{2}$ (Fig. 2, top and bottom), when they were not seen in the experiments in which the rare-earth isotopes were specifically studied.

b) By using the formulas of Ref. 7, the expected half-lives for such high energy $\alpha$ particles in the rare-earth region are very short, $2.0 \times 10^{-2} \mathrm{~s}$ for $5.14 \mathrm{MeV}$ in for instance ${ }^{148} \mathrm{Eu}, 5.6 \times 10^{-2} \mathrm{~s}$ for $5.27 \mathrm{MeV}$ in ${ }^{152} \mathrm{~Tb}$, and $2.4 \mathrm{~s}$ for $5.53 \mathrm{MeV}$ in ${ }^{172} \mathrm{Lu}$. (Eu, Tb and $\mathrm{Lu}$ are the chemical homologs of Bk, Es and Lr-No, respectively (Fig. 2)). The measured half-lives are longer by factors of $6 \times 10^{9}, 9.6 \times 10^{8}$ and $9.4 \times 10^{5}$, respectively. High spin isomers can explain such large retardations only if $\Delta \mathrm{L}_{\alpha}$ is equal to about 15 for the first and second cases, and about 12 for the third case 91. Taking into account the large availability of states with various spins in the rare-earth nuclei, far from closed shells, and the various possible decay modes, it is very difficult to see a situation where a state with at least such a high spin exists, and does not have any other way to decay, but by very long-lived $\alpha$ 's with $\Delta \mathrm{L}_{\alpha}$ values of 12 or 15 .

c) Another alternative could in principle be the existence, in rare-earth nuclei, of shape isomeric states, for instance long-lived isomeric states in the second minimum of the potential in the parent nuclei, which decay, by very retarded $\alpha$-particles, to the normal states of the daughter. (Such states have been discovered by us [11] in Os, Ir and $\mathrm{Hg}$ isotopes using the reaction between the deformed ${ }^{28} \mathrm{Si}$ and ${ }^{181} \mathrm{Ta}$ nuclei at bombarding energy below and around the Coulomb barrier). However, no super-deformed minima at low spins have been theoretically found in rare-earth nuclei with $\mathrm{Z} \geq 62$ and $\mathrm{N}<126$ [19]. Superdeformed minima have been produced at high spins in the $\mathrm{A} \sim 150$ region using heavy-ion reactions. This region has been studied very carefully. The minima are at high energies and the de-excitation is via multi-step or even single step $\gamma$-ray transitions [20]. It is very difficult to see how unknown high-spin isomeric states will be produced in the second minima via spallation reactions and be present in our sources, taking into account that high spins are not produced preferentially by such reactions, and that our sources were separated from nuclei with A 
150 , by a factor of about $1 \times 10^{5}$.

Since it is impossible to identify the observed $\alpha$-particle groups with any known activity in the whole nuclear chart, and, in addition, as shown above, they are not due to unknown long-lived isomeric states in the neutron-deficient $\mathrm{Yb}$ to $\mathrm{Pt}$ nuclei, nor due to unknown long-lived isomeric states in the rare-earth region, one may try to interpret them as due to some new unknown long-lived isomeric states in the actinide region. As mentioned in the introduction, the production of actinides via secondary reactions in the $\mathrm{W}$ target have been seen before [2]. ${ }^{236} \mathrm{Am}$ and ${ }^{236} \mathrm{Bk}$ (in long-lived isomeric states) have been produced and positively identified by the decay of their daughter, after $\beta^{+}$or EC decay(s), ${ }^{236} \mathrm{Pu}$ [2].

The measured energies of the $\alpha$-particle groups are low as compared to the known energies in the heavy actinides [21], and their lifetimes are enhanced by a factor of $10^{4}$ to $10^{7}$ as compared to the systematics [7] of $\alpha$-particle decay in this region of nuclei. From the identification of the isomeric states in the ${ }^{236} \mathrm{Am}$ and ${ }^{236} \mathrm{Bk}$ nuclei [2], it may be deduced that neutron-deficient actinide nuclei are produced via secondary reactions in the $\mathrm{W}$ target. Since the intensity of the $5.14 \mathrm{MeV}$ group in the Bk source grew at the beginning, one may conclude that this group is emitted from a daughter nucleus, produced by the $\beta^{+}$or EC process, rather than from an isotope of Bk itself. (One may exclude $\alpha$-particle decay from the produced Bk isotope to the daughter nucleus which decays by the $5.14 \mathrm{MeV}$ group, since no group of $\alpha$-particles was seen in the first measured spectrum, taken about two and a half years before the one seen in fig. 3 (ref. [2], fig. 1c).

Tables I and II contain measured and calculated $\alpha$-decay energies and lifetimes which we use to identify those nuclides emitting these unidentified $\alpha$-groups. Curium and Americium are the immediate $\beta^{+} / \mathrm{EC}$ decay products of Berkelium. In column 1 of table 1 the $\alpha$-particle energies [21] for the ground state to ground state transitions of several neutron-deficient $\mathrm{Cm}$ and Am nuclei are given. These energies of 5.6 to 6.5 MeV (some from experiment and some from systematics), are substantially higher than the observed $5.14 \mathrm{MeV}$. It is seen in table 2 that the half-life of $3.8 \mathrm{y}$ is enhanced by a factor of $1.5 \times 10^{5}$ or $3.8 \times 10^{4}$ as compared to the calculated values [7] for such energy from two typical parent nuclei, ${ }^{238} \mathrm{Am}$ and ${ }^{238} \mathrm{Cm}$, respectively.

It is also shown in column 2 of table 1 that the ground state to ground state transition energies (mostly from systematics) in neutron-deficient Es isotopes are in the range of 7.4 to $8.2 \mathrm{MeV}$. These energies are much higher than the observed 5.27 MeV. Furthermore, as seen in table 2, a half-life of $625 \mathrm{~d}$ for such low energy $\alpha$-particles in Es isotopes is too fast by about a factor of $2.8 \times 10^{6}$.

The typical ground state to ground state $\alpha$-energies for Lr-No isotopes are $8-9 \mathrm{MeV}$ and the observed $5.53 \mathrm{MeV}$ is very low compared to these values. (For a reason which will become clear in sec. III.c, $\alpha$-energies for Fm isotopes rather than for Lr and No are given in table 1). Also, a half-life of $26 \mathrm{~d}$ for $5.53 \mathrm{MeV} \alpha$-particles in the Lr-No region, is enhanced by a factor of about $8.5 \times 10^{6}$ (see table 2 ).

From this discussion it is obvious that previously unknown isomeric states, rather than the normal ground states, were produced in the reaction. As mentioned in the introduction, long-lived isomeric states have recently been observed in the second [9 11] and third [11] wells of the potential energy surfaces. In what follows, the unidentified $\alpha$-groups are analyzed in terms of production of such isomeric states. 
In table 1, predicted [12] $\alpha$-particle energies are given for several neutron-deficient actinide nuclei, for transitions from the second minima in the parent nuclei to the ground states and to the second and third minima in the daughters, and from the third minima in the parents to the third and second minima in the daughters. Table 2 gives calculated [9] half-lives for various transitions from the second and the third minima in the parents, to the corresponding minima in the daughters. The effect of large deformations on the $\alpha$-decay was calculated with the potential parameters of Igo [22] but with a deformed radius $\mathrm{R}(\theta)=\mathrm{c}(\beta) \mathrm{R}_{0}\left[1+\beta_{2} \mathrm{Y}_{20}(\theta)+\beta_{3} \mathrm{Y}_{30}(\theta)+\beta_{4} \mathrm{Y}_{40}(\theta)\right]$ where $\mathrm{c}(\beta)$ is determined from the volume conservation condition and $\mathrm{R}_{0}=\mathrm{r}_{0} \times \mathrm{A}^{1 / 3}$. A formation probability for the $\alpha$-particle of [23] 25(4/A) ${ }^{3}$ was used in the calculations. Only the decay out of the tip $(\theta=0)$ of the emitting nucleus was directly calculated. Solid angle averaging increases the lifetimes by about a factor of 3. A $4 \%$ increase in the radius parameter (from $\mathrm{r}_{0}=1.17$ to $\mathrm{r}_{0}=1.22 \mathrm{fm}$ ), reasonable for $\alpha$-decay from a highly excited isomeric state, reduces the lifetimes by about a factor of $4 . \beta_{2}$ and $\beta_{4}$ values for the different potential minima were deduced from the $\epsilon_{2}$ and $\epsilon_{4}$ values given in ref. [12], using fig. 2 of ref. [24]. For third minima transitions, where $\epsilon_{2}$ and $\epsilon_{3}$ values are given in ref. [12], three calculations were performed. In the first one a $\beta_{2}$ value was obtained as described above and $\beta_{3}$ was taken as zero. In the second calculation $\beta_{3}$ was taken equal to $\epsilon_{3}$, and in the third calculation the parameters obtained by Ćwiok et al. [25] for the hyperdeformed state in ${ }^{232} \mathrm{Th}$ of $\beta_{2}=0.85, \beta_{3}=0.35$ and $\beta_{4}=0.18$, were used.

\section{A. The 5.14 MeV $\alpha$-Particle Group in the Bk Source}

It is seen in table 1 that from the energy point of view the $5.14 \mathrm{MeV} \alpha$-particle group may correspond to a $\mathrm{II}^{\text {min }}$ to $\mathrm{II}^{\text {min }}$ transition from ${ }^{238} \mathrm{Am}$ or ${ }^{239} \mathrm{Am}$, or to a III ${ }^{\text {min }}$ to $\mathrm{III}^{\text {min }}$ transition from ${ }^{238} \mathrm{Cm},{ }^{239} \mathrm{Cm}$ or ${ }^{237} \mathrm{Am}$. Table 2 shows that the observed lifetime is consistent with a II ${ }^{\text {min }}$ to $\mathrm{II}^{\mathrm{min}}$ transition. It is also consistent with a III ${ }^{\mathrm{min}}$ to $\mathrm{III}^{\mathrm{min}}$ transition, if $\beta_{3}$ $=0$. However, the observed half-life is retarded by 1 to 3 orders of magnitude if various octupole deformations are considered. It is seen in fig. 6 that according to the predictions of Howard and Möller [12 the second minimum in ${ }^{238} \mathrm{Am}$ is more pronounced than the third minimum in the same nucleus or in ${ }^{238} \mathrm{Cm}$ which favors the $\mathrm{II}^{\text {min }}$ to $\mathrm{II}^{\text {min }}$ transition. In conclusion, the $5.14 \mathrm{MeV}$ group seen in the Bk source may be explained by an unhindered $\mathrm{II}^{\text {min }}$ to II ${ }^{\text {min }}$ transition from ${ }^{238} \mathrm{Am}$ or ${ }^{239} \mathrm{Am}$. However, the possibility that it is due to a hindered III ${ }^{\text {min }}$ to $\mathrm{III}^{\text {min }}$ transition from ${ }^{238} \mathrm{Cm},{ }^{239} \mathrm{Cm}$ or ${ }^{237} \mathrm{Am}$ can not be ruled out. This is so because retardation of $\alpha$-particle decay may be due to small overlap between the initial and final wave functions, even for transitions between states which belong to the same potential well. Furthermore, the third minimum in nuclei around ${ }^{238} \mathrm{Cm}$ may perhaps be more pronounced than seen in fig. 6 if high spins are considered.f

\footnotetext{
${ }^{4}$ Such a phenomenon is known in the second well in nuclei like for instance ${ }^{236} \mathrm{Pu}$ or ${ }^{242} \mathrm{Cm}$ where two shape isomeric states have been observed, and in fact the higher excited state lives longer than the lower one. (D. N. Poenaru, M. S. Ivaşcu, and D. Mazilu in Particle Emission from Nuclei, Eds. D. N. Poenaru and M. S. Ivaşcu, CRC Press, Vol. III, p. 41 (1989).
} 


\section{B. The 5.27 MeV $\alpha$-Particle Group in the Es Source}

Let us now discuss the $5.27 \mathrm{MeV}$ group seen in the Es source. As seen in table 1, columns 6 and 7 , it is consistent, from the energy point of view, with a III $^{\text {min }}$ to III $^{\text {min }}$ transition or III ${ }^{\text {min }}$ to $\mathrm{II}^{\text {min }}$ transition, from ${ }^{246} \mathrm{Es}$ or ${ }^{247} \mathrm{Es}$. It is seen in table 2 that the measured half-life is enhanced by about a factor of 70 as compared to the calculated value, for a $\mathrm{II}^{\mathrm{min}}$ to II $^{\text {min }}$ transition. In a III $^{\text {min }}$ to II $^{\text {min }}$ transition the $\alpha$-particle sees the potential of the daughter nucleus in the second minimum. Because of the change in the shape in a transition from the $\mathrm{III}^{\text {min }}$ to the $\mathrm{II}^{\text {min }}$, such a transition will be hindered as compared to a $\mathrm{II}^{\text {min }}$ to $\mathrm{II}^{\text {min }}$ transition. (In the case of the $8.6 \mathrm{MeV} \mathrm{III}^{\text {min }}$ to $\mathrm{II}^{\text {min }} \alpha$-transition from ${ }^{195} \mathrm{Hg}$ [11], the hindrance factor as compared to a $\mathrm{II}^{\text {min }}$ to $\mathrm{II}^{\text {min }}$ transition is in the range of $\left.10^{16}\right)$. This means that the enhancement factor for the lifetime of the $5.27 \mathrm{MeV}$ assuming III $^{\text {min }}$ to II ${ }^{\text {min }}$ transition, should be much larger than 70. But it is quite difficult to understand such a large enhancement and it is much more reasonable to assume that this group is due to a $\mathrm{III}^{\text {min }}$ to III $^{\text {min }}$ transition. Considering a III ${ }^{\text {min }}$ to III $^{\text {min }}$ transition, it is seen in table 2 that the measured half-life is enhanced by a factor of 20 if $\beta_{3}=0$, and it is retarded by a factor of $1.6-120$ if $\beta_{3}$ and also $\beta_{4}$ are included in the calculations. Since it is easier to understand retardation of $\alpha$-decay rather than enhancement, in transitions within the same potential well, it seems that a quadrupole deformation alone is not enough to describe the hyperdeformed states in the daughter nuclei around ${ }^{243} \mathrm{Bk}$.

Fig. 6 shows 12 that in the second minimum of ${ }^{247}$ Es the outer barrier is quite low, while for the third minimum the inner barrier is very large, and in fact the third minimum is predicted to be the true ground state of this nucleus, being $0.61 \mathrm{MeV}$ below the normal, low deformed, ground state [12]. Unfortunately there are no predictions in this case for the potential at even larger deformations, beyond the third minimum. (In the case of ${ }^{232} \mathrm{Th}$ 25 the outer barrier in the third minimum is quite high).

\section{The 5.53 MeV $\alpha$-Particle Group in the Lr-No Source}

To the best of our knowledge there are no predictions for the second and third minima for Lr-No nuclei. Therefore the predictions [12 for neutron-deficient Fm isotopes were used in deducing the various $\alpha$-energies given in table 1 . It is seen that such a low energy as $5.53 \mathrm{MeV}$ may only be due to an isomeric state in the third well of the potential. From the energy point of view it may be either due to a III ${ }^{\text {min }}$ to III ${ }^{\text {min }}$ or a III ${ }^{\text {min }}$ to II $^{\text {min }}$ transition. By extrapolating according to the $\alpha$-energies from ${ }^{246} \mathrm{Es}$ and ${ }^{248} \mathrm{Fm}$, one finds the parent isotope around ${ }^{252}$ No or ${ }^{254} \mathrm{Lr}$. It is seen in table 2 that the measured half-life of 26 days is enhanced by a factor of about 160 as compared to the prediction assuming a $\mathrm{II}^{\mathrm{min}}$ to $\mathrm{II}^{\mathrm{min}}$ transition. Based on the arguments given above one may conclude that the $5.53 \mathrm{MeV}$ group is due to a III ${ }^{\text {min }}$ to III $^{\text {min }}$ and not due to a III ${ }^{\text {min }}$ to II $^{\text {min }}$ transition. Regarding a III ${ }^{\text {min }}$ to III $^{\text {min }}$ transition, it is seen in table 2 that the measured half-life is still enhanced by a factor of about 85 if only $\beta_{2}$ deformation is taken into account in the calculations. However, a consistency is obtained when $\beta_{3}$ or $\beta_{3}$ plus $\beta_{4}$ are included. Fig. 6 shows that the features mentioned above in ${ }^{247} \mathrm{Es}$ occur also in ${ }^{248} \mathrm{Fm}$. The outer barrier in the second minimum is low and the inner barrier in the third minimum is high, and the third minimum is in fact predicted [12] to be the true ground state of this nucleus, being $1.76 \mathrm{MeV}$ below the ground 
state of the first minimum. One may assume that the potential-energy curves of the nuclei around ${ }^{252} \mathrm{No}-{ }^{254} \mathrm{Lr}$ are similar, explaining the observation of the $5.53 \mathrm{MeV}$ group in the Lr-No source.

Table 3 summarizes the half-lives of the isomeric states as compared to the half-lives of their corresponding normal-deformed ground states. It is assumed in this comparison that the 5.14, 5.27 and 5.53 MeV groups are from ${ }^{238} \mathrm{Am},{ }^{247} \mathrm{Es}$ and ${ }^{252} \mathrm{No}$ nuclei, respectively (see table 1). For completeness the data on the isomeric states in ${ }^{236} \mathrm{Am}$ and ${ }^{236} \mathrm{Bk}$ [2] are also included in this table. It is seen that the lifetimes of the isomeric states are $10^{4}$ to $10^{6}$ longer as compared to their corresponding ground states of normal deformations.

\section{Production Cross Sections of the Various Isotopes}

As mentioned in the introduction the production yield in the secondary reactions depends on two cross sections: a) the cross section for production of suitable fragments with high enough kinetic energies, which may interact with other $\mathrm{W}$ nuclei in the target, and b) the heavy-ion reaction cross section between such fragments and other $\mathrm{W}$ nuclei in the target. In the following we will try to deduce the cross sections for the second reaction, based on reasonable estimates about the first step of the reaction, the production of the high-energy fragment.

Table 4 summarizes the process of deducing the heavy-ion reaction cross sections. The data regarding the production of the isomeric states in ${ }^{236} \mathrm{Am}$ and ${ }^{236} \mathrm{Bk}$ [2] are also included in this table. The numbers of produced atoms, which were deduced from the measured intensities and half-lives of the $\alpha$-lines, are given in column 2 . One can see that $3 \times 10^{5}$ to $1.4 \times 10^{4}$ atoms were produced in the isomeric states of the various isotopes. Column 3 gives the total cross sections which are in the $10^{-12}$ to $10^{-14} \mathrm{~b}$ region. These cross sections were deduced from the number of produced nuclei, taking into account the integrated number of protons in the beam and the thickness of the target. In column 4 the most suitable possible reactions are listed assuming that the bombarding energies of the relevant secondary fragments are around the Coulomb barriers between the projectile and target nuclei, at 4.2 to $4.8 \mathrm{MeV} /$ nucleon. It is seen in column 5 that in general, at $\mathrm{E}_{L a b}$ equal to the Coulomb barrier, the reactions are quite hot with excitation energies of the compound nuclei around $30-40 \mathrm{MeV}$. The yield from higher bombarding energies is presumably smaller since first, the production of high energy fragments is going down as their outgoing energy increases [17], and secondly, producing the compound nucleus at higher excitation energy results in a lower evaporation residue cross section, because of the competition with fission during the cooling process. Only reactions with evaporation of a proton, an $\alpha$-particle, or a proton

\footnotetext{
${ }^{5}$ It should be mentioned that in addition to various theoretical predictions about the existence of the third minimum in the potential energy in various actinide nuclei which include refs. [12] and [19] and which was first proposed by P. Möller and J.R. Nix (Proc. Int. Conf. on Physics and Chemistry of Fission, Rochester, 1073 (IAEA, Vienna 1974) Vol. I, p. 103), experimental evidences were also obtained in Th and U nuclei by J. Blons et al. (Nucl. Phys. A477, 231 (1988)), and A. Krasznahorkay et al. (APH N.S., Heavy Ion Physics 7, 35 (1998)).
} 
+ a neutron and an $\alpha$-particle + a neutron were considered. (The yields from the (HI,xn) reactions, in particular from the (HI,3n) reactions, are expected to be less important: first, higher neutron-excess fragments which act as projectiles are needed, and these are produced less easily; and secondly, one loses more yield, due to competition with fission, in three step evaporation processes as compared to one or two stepsi). The same cross sections were assumed for the various heavy-ion reactions which contribute to the production of a particular isotope. The range of the total production cross sections of the various fragments listed in column 4, are given in column 6 . These cross sections were estimated using first the measured cross sections for the production of various Sc isotopes in the interaction of 18.2 $\mathrm{GeV}$ protons on a Ta target [26]. (These values are very similar to those obtained in the interaction of $28.5 \mathrm{GeV}$ protons on $\mathrm{Pb}[27]$ ). Secondly, by using exponential extrapolation, values for heavier Sc isotopes were obtained. The cross sections for the various fragments considered in table 4 were then obtained using A/Z scaling. Column 7 in table 4 gives the values of " $\mathrm{f}$ ", the ratio of the production cross sections for high energy fragments, above or near the Coulomb barrier, to the total cross sections of the corresponding fragments. It was assumed to be $1 \%$ for the fragments which produced the ${ }^{236} \mathrm{Am}$ isotope, and reduced exponentially to $0.1 \%$ for those which produced the ${ }^{252}$ No isotope. (A cross section of about $20 \mu \mathrm{b}$ is deduced for $\mathrm{Ar}$ ions of $\geq 4.5 \mathrm{MeV} /$ nucleon produced in the interaction of $5.5-\mathrm{GeV}$ protons with $\mathrm{U}$ (fig. 14 of ref. [17]). Therefore, cross sections of about $8 \mu \mathrm{b}$ or $0.9 \mu \mathrm{b}$ for the production of high energy ${ }^{51} \mathrm{Ti}$ or ${ }^{67} \mathrm{Cu}$ ions respectively, in the interaction of $24-\mathrm{GeV}$ protons with $\mathrm{W}$, are reasonable).

The last column in table 4 gives the deduced evaporation residue cross sections which are from 0.2 to $13 \mathrm{mb}$. The results depend somewhat on the assumptions made above. However, it seems that first, the cross sections do not decrease when the Z-value of the evaporated residual nucleus increases from 95 to 102, and secondly, the cross sections are very large as compared to known heavy-ion reactions, in particular when ${ }^{252}$ No is considered, where the typical cross sections are below $1 \mu \mathrm{b}$ [8].

Fig. 7 shows calculated fusion cross sections using the coupled-channel deformed fusion code of Ref. [28] for a typical ${ }^{70} \mathrm{Zn}+{ }^{186} \mathrm{~W}$ reaction. Curve $7 \mathrm{a}$ was obtained assuming that the projectile and the target nuclei are spherical. In curve $7 \mathrm{~b}$ the known [29] quadrupole deformation of the target nucleus was included in the calculations. Curve $7 \mathrm{c}$ displays the results when coupling to the $2^{+}$and $3^{-}$vibrational states of the projectile nucleus were also taken into account. In the secondary reaction experiments, the projectile is a fragment which had been produced only within about $2 \times 10^{-14} \mathrm{~s}$ before interacting with another $\mathrm{W}$ nucleus in the target. During this short time it is still at high excitation energy and quite deformed. In particular, since it is produced as a deformed fragment, it may have a deformation which is typical to the second or third well of the potential, rather than to normal deformation. Curve $7 \mathrm{~d}$ shows the results of calculations taking into account the known quadrupole deformation of the target nucleus, and assuming for the projectile fragment $\left({ }^{70} \mathrm{Zn}\right)$ a $\beta_{2}$ value of

\footnotetext{
${ }^{6}$ For instance, at $\mathrm{E}_{L a b}=154 \mathrm{MeV}\left(\mathrm{E}_{x}(\mathrm{C} . \mathrm{N})=.51.9 \mathrm{MeV}\right)$, cross sections of about 70 and $37 \mu \mathrm{b}$ were obtained for the ${ }^{175} \mathrm{Lu}\left({ }^{32} \mathrm{~S}, \mathrm{p} 2 \mathrm{n}\right){ }^{204} \mathrm{Rn}$ and the ${ }^{175} \mathrm{Lu}\left({ }^{32} \mathrm{~S}, \alpha 2 \mathrm{n}\right){ }^{201}$ At reactions respectively, as compared to about $7 \mu \mathrm{b}$ for the ${ }^{175} \mathrm{Lu}\left({ }^{32} \mathrm{~S}, 4 \mathrm{n}\right){ }^{203} \mathrm{Fr}$ and ${ }^{175} \mathrm{Lu}\left({ }^{32} \mathrm{~S}, 3 \mathrm{n}\right){ }^{204} \mathrm{Fr}$ reactions (S. Gelberg, Ph. D. thesis, 1991).
} 
0.6, a somewhat higher value than typical for the second minimum of the potential in this region of nuclei [30]. According to curve $7 \mathrm{a}$, a cross section of about $10 \mathrm{mb}$ is predicted at bombarding energies around the Coulomb barrier of $232 \mathrm{MeV}$. This result can not explain the experimental values, since one would fall considerably below the measured evaporation residue cross sections, allowing for competition with fission in producing the final evaporation residual nuclei. (One gets larger fusion cross sections at higher bombarding energies, but than the number of evaporated particles increases, which will decrease the evaporation residue production cross section). In curves $7 \mathrm{~b}$ and $7 \mathrm{c}$, fusion cross sections of $60-$ $70 \mathrm{mb}$ are predicted at bombarding energies around the Coulomb barrier. An even larger fusion cross section of about $160 \mathrm{mb}$ is predicted (curve $7 \mathrm{~d}$ ) when a large deformation for the fragment projectile is assumed. These predictions are consistent with the experimental results if $\Gamma_{n} / \Gamma_{f}, \Gamma_{p} / \Gamma_{f}$ and $\Gamma_{\alpha} / \Gamma_{f}$ are not too small. It is also seen in curve $7 \mathrm{~d}$ that the very cold fusion reaction, i.e. the radiative capture reaction at $\mathrm{E}_{c . m}$. $\sim 215 \mathrm{MeV}$, below the one neutron separation energy where the competition with fission is very much reduced, is perhaps possible with a fusion cross section around $30 \mathrm{mb}$. (If one would have assumed only this reaction as compared to the four reactions ( $\mathrm{p}$, pn, $\alpha$ and $\alpha \mathrm{n}$ ) considered in table 4 , a four times larger cross sections than those given in the last column of table 4 are needed in order to be consistent with the experimental results). Thus, the unusually large evaporation residue cross sections deduced from the secondary reaction experiments, can consistently be understood, provided that very little extra-push energy 31 33] is needed in order to produce the compound nucleus. An extra-push energy means that one has to shift the plots of fig. 7 to the right by its value. It is seen in curves $7 \mathrm{~b}$ and $7 \mathrm{c}$ that around $232 \mathrm{MeV}$, an extrapush energy of $5 \mathrm{MeV}$ reduces the fusion cross sections down to $25-35 \mathrm{mb}$. This is pretty low, remembering that one or two particles have to be evaporated, and still leave around $10 \mathrm{mb}$ for the production cross section of the residual nucleus. For curve $7 \mathrm{~d}$ zero extra-push energy is consistent with the data for bombarding energies around $215 \mathrm{MeV}$, while around $232 \mathrm{MeV}$, an extra-push energy around $10 \mathrm{MeV}$ is tolerable. It should be mentioned that extra-push energies of about 300 [31], 50 [32] and $20 \mathrm{MeV}$ [33] were predicted for the ${ }^{70} \mathrm{Zn}$ $+{ }^{186} \mathrm{~W}$ reaction. The small experimentally deduced extra-push values, of $0-10 \mathrm{MeV}$, are consistent with the previous conclusion that the compound nucleus was formed in the second or third well of the potential, and not in the innermost well.

Fig. 8 shows several calculated shapes of the compound nuclei, using Eq. (1) for the deformed radii and various values for the deformation parameters. The calculated shapes of the target and corresponding projectile nuclei are also given in the figure. In fig. 8 (top) two calculated shapes for the compound nucleus with $\mathrm{A}=239$ are displayed. The first one (left) shows the calculated shape of the compound nucleus with deformation parameters [12] of the normal ground state, while in the second one (right) the deformation parameters 12 of the second minimum were used in the calculations. Three calculated shapes for $\mathrm{A}=253$ are seen in fig. 8 (bottom). In the left side the parameters of the normal ground state were used in the calculation, while in the middle only the parameter $\beta_{2}$ of ref. [12 for the third minimum of the potential was used. In the right (bottom) side of fig. 8 the deformation parameters for the hyperdeformed minimum in ${ }^{232} \mathrm{Th}$ 25 were taken into account in our calculations. It is qualitatively seen that much less rearrangement and matter penetration accompanied by dissipation, and hence much less extra-push energy, is needed to form the compound nucleus in the second and particularly in the third well of the potential, compared 
to formation in the normal states.

\section{E. Consequences Regarding the Production of the Long-Lived Superheavy Element with $\mathrm{Z}=112$}

The existence of the long-lived isomeric states in the second and third wells of the potential can explain, in a very consistent manner [34], the discovery [3] -5] of the superheavy element with $\mathrm{Z}=112$. The experimental evidence for the existence of this element was based mainly on the observation of fission fragments in $\mathrm{Hg}$ sources which were separated from the CERN W target [3], and on the mass measurements of the fissioning nuclei, which were interpreted as due to five different molecules of the isotope with $\mathrm{Z}=112$ and $\mathrm{N} \cong$ 160 [5]. While it was impossible to disprove the data it seemed very difficult to understand them [35], in particular the long lifetime of the observed activities of several weeks, and the large deduced heavy ion reaction cross section of a few mb. It was pointed out [5] that the radiative capture processes like ${ }^{184} \mathrm{~W}\left({ }^{88} \mathrm{Sr}, \gamma\right){ }^{272} 112$ or ${ }^{186} \mathrm{~W}\left({ }^{86} \mathrm{Sr}, \gamma\right){ }^{272} 112$ are possible at bombarding energies around the Coulomb barrier. Such reactions in principle could yield large evaporation residue cross sections. However, it was thought that the large extra-push energy expected for such reactions will drastically reduce the fusion cross section.

The existence of the long-lived isomeric states in the second and third wells of the potential, and the relatively very little extra-push energy required for their production, provide a consistent interpretation to the experimental observations. The long lifetime of several weeks shows that the ${ }^{\sim 272} 112$ nucleus was probably produced in a long-lived isomeric state rather than in the normal ground state where a half-life of about $240 \mu$ s was observed for the ${ }^{277} 112$ isotope [36]. The large deduced fusion cross section of a few $\mathrm{mb}$ ( [5] and the

discussion below), as compared to the evaporation residue cross section of about $1 \mathrm{pb}$ for the production of ${ }^{277} 112$ [36], strongly indicates that the isomeric state is in the second or third well of the potential, rather than in the first normal-deformed well. (The observation of the isomeric states in the third well, which may actually be the true ground states in the very heavy Es and Lr-No nuclei, may imply a similar situation also in the superheavy nuclei region around $\mathrm{Z}=112$ ). In table 4 a cross section of $3.8 \mathrm{mb}$ for the second step of the reaction is deduced from the data, assuming a contribution from the radiative capture process alone. (Adding contributions from other reactions like $\left({ }^{x} \mathrm{Sr}, \mathrm{n}\right),\left({ }^{x} \mathrm{Y}, \mathrm{p}\right)$ or $\left({ }^{x} \mathrm{Zr}, \alpha\right)$, will decrease the deduced cross section). This value is about the same as those obtained in the actinides. However, in the actinides, relatively hot reactions took place, while in the $\mathrm{Sr}+\mathrm{W}$ reactions an extremely cold type of reaction, the radiative capture with excitation energies of the compound nuclei of $2.0-10.0 \mathrm{MeV}$ (table 4), is possible. The lower fusion cross section in the superheavy element region of about $4 \mathrm{mb}$ as compared to the predicted $60-160 \mathrm{mb}$ in the actinides (see above), may be due to a larger extra-push energy needed in the superheavies as compared to the actinides. It was shown that extra-push energies around $30-40 \mathrm{MeV}$ are consistent with the measured fusion cross section of about $4 \mathrm{mb}$, assuming various deformations (figs. 4 and 5 of ref. [34]), or deformations which are typical to the second minimum of the potential (fig. 3 of ref. [37]), for the secondary Sr fragment. An extra-push energy of $30-40 \mathrm{MeV}$ is small compared to the predictions of $\gg 300$ [31] and $\gg 50$ [32] made for the production of the compound nucleus in the normal states. (It is compatible with an extra-push value of $\sim 40 \mathrm{MeV}$ predicted for this reaction in Ref. [33]). 
It is in accord with the values of $0-10 \mathrm{MeV}$ obtained above in the actinides for producing the compound nucleus in the second or third well of the potential-energy surfaces. Thus, the combined effect of first, producing the compound nucleus in the third or second well of the potential, which requires much lower extra-push energy as compared to its production in the normal states, and secondly, the reduced Coulomb repulsion between a $\mathrm{W}$ target nucleus and a deformed fragment (produced in the secondary reaction within about $2 \times 10^{-14}$ $\mathrm{s}$ before interacting with the $\mathrm{W}$ target), as compared to the Coulomb repulsion caused by the interaction with a normal projectile, enables one to understand the large fusion cross section, even if an extra-push energy of $30-40 \mathrm{MeV}$ were required in the process.

\section{SUMMARY}

The problems of the low energies, large lifetime enhancements and high production cross sections of the $\alpha$-particle groups of 5.14, 5.27 and $5.53 \mathrm{MeV}$, seen in the Bk, Es and Lr-No sources, respectively, have consistently been interpreted as due to formation of long-lived isomeric states in the second and third wells of the potential-energy surfaces. The low energies fit well with theoretical predictions [12] for $\mathrm{II}^{\mathrm{min}}$ to $\mathrm{II}^{\mathrm{min}}$ (or III ${ }^{\mathrm{min}}$ to III ${ }^{\mathrm{min}}$ ) transition in the first case, and to III ${ }^{\mathrm{min}}$ to $\mathrm{III}^{\mathrm{min}}$ transitions in the second and third cases. The large enhancements of the $\alpha$-particle decays are due to the increased penetrabilities through the barriers of superdeformed or hyperdeformed nuclei. The large production cross sections are due to two effects: a) the rather little, if any, extra-push energy needed to produce the compound nucleus in the second or third well of the potential-energy surfaces. b) the increased fusion cross section due to the deformation of the projectile (fragment) in the secondary reactions, as compared to normal projectiles. This increased fusion cross section can explain the observed production of the evaporation residue nuclei, even for an extra-push energy up to $10 \mathrm{MeV}$. According to theoretical predictions [12], the isomeric states in the third well in the Es and Lr-No cases, may actually be the true (or nearby the true) ground states of the particular nuclei.

The existence of long-lived isomeric states in the second and third wells of the potentialenergy surfaces seems to be a general phenomenon in heavy nuclei. They have been seen in neutron-deficient nuclei from the Os - Hg region [11], through Fr [9, 10], and up to the heavy actinide nuclei, like Am, Bk, Es and Lr-No. In addition to their very long lifetimes, much longer than that of their corresponding normal-deformed ground states, they may decay by very enhanced $\alpha$-particle emission, in $\mathrm{II}^{\mathrm{min}}$ to $\mathrm{II}^{\mathrm{min}}$ [9] or $\mathrm{III}^{\mathrm{min}}$ to $\mathrm{III}^{\mathrm{min}}$ (the present work) transitions, or by very retarded $\alpha$-particle decay, in II ${ }^{\text {min }}$ to I ${ }^{\text {min }}$ or in III ${ }^{\text {min }}$ to II ${ }^{\text {min }}$ transitions [11], and also by long-lived proton radioactivity [10].

It was shown that the existence of the isomeric states in the second and third well of the potential-energy surfaces enables one to understand, in a fully consistent manner, the production of the long-lived superheavy element with $\mathrm{Z}=112$ [3 5, 34], much longer than seen in Ref. [36]. In general the discovery of such isomeric states with their very unusual decay properties, the very much reduced extra-push energy needed for their production in interactions between very heavy ions, and the possibility that the third minimum is actually the true ground state of very heavy and perhaps superheavy nuclei, provide completely new considerations in the study of heavy and superheavy elements. 


\section{ACKNOWLEDGEMENTS}

We are grateful to C. J. Batty, A. I. Kilvington, G. W. A. Newton, J. D. Hemingway and S. Eshhar for taking part in the first stages of the experiments. The late A. M. Friedman and V. J. Robinson also participated at the beginning of the experiments. We appreciate very much the valuable discussions with $\mathrm{N}$. Zeldes and the technical assistance of S. Gorni, O. Skala and the electronic team of the Hebrew University. D. K. acknowledges the financial support of the DFG. 


\section{REFERENCES}

[1] A. Marinov, S. Eshhar, and J. L. Weil, Proc. Inter. Symp. on Superheavy Elements, Lubbock, Texas, 1978, edited by M. H. K. Lodhi (Pergamon, New York, 1978), p. 72.

[2] A. Marinov, S. Eshhar, and D. Kolb, Phys. Lett. B191, 36 (1987).

[3] A. Marinov, C. J. Batty, A. I. Kilvington, G. W. A. Newton, V. J. Robinson, and J. D. Hemingway, Nature 229, 464 (1971).

[4] A. Marinov, C. J. Batty, A. I. Kilvington, J. L. Weil, A. M. Friedman, G. W. A. Newton, V. J. Robinson, J. D. Hemingway, and D. S. Mather, Nature 234, 212 (1971).

[5] A. Marinov, S. Eshhar, J. L. Weil, and D. Kolb, Phys. Rev. Lett. 52, 2209 (1984); 53, $1120(\mathrm{E})(1984)$.

[6] A. Marinov, S. Eshhar, and D. Kolb, Fizika 19, Supplement 1, 67 (1987).

[7] V. E. Viola Jr. and G. T. Seaborg, J. Inorg. Nucl. Chem. 28, 741 (1966).

[8] S. Hofmann, VI Int. School-Seminar on Heavy Ion Physics, Dubna, Russia, 1997, Eds. Yu. Ts. Oganessian and R. Kalpakchieva, World Scientific, p. 385.

[9] A. Marinov, S. Gelberg, and D. Kolb, Mod. Phys. Lett. A11, 861 (1996).

[10] A. Marinov, S. Gelberg, and D. Kolb, Mod. Phys. Lett. A11. 949 (1996).

[11] A. Marinov, S. Gelberg, and D. Kolb, preprint, arXiv:nucl-ex/0006012.

[12] W. M. Howard and P. Möller, At. Data Nucl. Data Tables 25, 219 (1980).

[13] G. H. Higgins, The Radiochemistry of the Transcurium Elements, NAS-NS 3031 (1960).

[14] G. W. A. Newton, V. J. Robinson, M. Skarestad, and J. D. Hemingway, J. Inorg. Nucl. Chem. 35, 1435 (1973).

[15] J. R. Grover, Phys. Rev. 126, 1540 (1962).

[16] G. Rudstam and G. Sørenson, J. Inorg. Nucl. Chem., 28, 771 (1966).

[17] A. M. Poskanzer, G. W. Butler, and E. K. Hyde, Phys. Rev. C 3, 882 (1971).

[18] B. Neidhart and K. Bächmann, J. Inorg. Nucl. Chem., 33, 3227 (1971).

[19] S. J. Krieger, P. Bonche, M. S. Weiss, J. Meyer, H. Flocard, and P.-H. Heenen, Nucl. Phys. A542, 43 (1992).

[20] A. Ataç et al., Z. Phys. A, 355, 343 (1996)).

[21] G. Audi, O. Bersillon, J. Blachot, and A. H. Wapstra, Nucl. Phys. A624, 1 (1997).

[22] G. Igo, Phys. Rev. Lett. 1, 72 (1958).

[23] H. Enge, Introduction to Nuclear Physics (Addison-Wesley, 1971).

[24] W. Nazarewicz and I. Ragnarsson, Handbook of Nuclear Properties, Eds. D. Poenaru and W. Greiner, Clarendon Press, Oxford, p. 80 (1996).

[25] S. Ćwiok, W. Nazarewicz, J. X. Saladin, W. Plóciennik, and A. Johnson, Phys. Lett. B 322, 304 (1994).

[26] E. Hagebø and H. Ravn, J. Inorg. Nucl. Chem. 31, 897 (1969).

[27] Y. Y. Chu, G. Friedlander, and L. Husain, Phys. Rev. C15, 352 (1977).

[28] J. Fernández-Neillo, C. H. Dasso, and S. Landowne, Code CCDEF, Comp. Phys. Comm. 55, 409 (1985).

[29] S. Raman, C. H. Malarkay, W. T. Miller, C. W. Nestor, Jr., and P. H. Stelson, At. Data Nucl. Data Tables 36, 1 (1987).

[30] M. Girod, J. P. Delaroche, D. Gogny, and J. P. Berger, Phys. Rev. Lett. 62, 2452 (1989).

[31] J. P. Blocki, H. Feldmeier, and W. J. Swiatecki, Nucl. Phys. A459, 145 (1986).

[32] J. R. Nix and A. J. Sierk, Proc. $6^{\text {th }}$ Adriatic Int. Conf. Nucl. Phys., Frontiers of Heavy- 
Ion Physics, Eds. N. Cindro, W. Greiner and R. Ĉaplar, (World Scientific, 1987), p. 333.

[33] P. Fröbrich, Phys. Lett. B215, 36 (1988).

[34] A. Marinov, S. Gelberg, and D. Kolb, in $6^{\text {th }}$ Int. Conf. on Nuclei Far from Stability and $9^{\text {th }}$ Int. Conf. on Atomic Masses and Fundamental Constants, Bernkastel-Kues, Germany, Ins. Phys. Conf. Ser. No. 132, p. 437 (1992).

[35] R. C. Barber, N. N. Greenwood, A. Z. Hrynkiewicz, Y. P. Jeannin, M. Lefort, M. Sakai, I. Ulehla, A. H. Wapstra, and D. H. Wilkinson, Proc. Part. Nucl. Phys. 29, 453 (1992).

[36] S. Hofmann, V. Ninov, F. P. Heßberger, P. Armbruster, H. Folger, G. Münzenberg, H. J. Schött, A. G. Popeko, A. V. Yeremin, S. Saro, R. Janik, and M. Leino, Z. Phys. A 354, 229 (1996).

[37] A. Marinov, S. Gelberg, and D. Kolb, Proc. Int. Sym. on Exotic Nuclear Shapes, Debrecen, Hungary, (1997), Eds. Zs. Dombrádi, Z. Gácsi, and A. Krasznahorkay, APH N.S., Heavy Ion Physics 7, 47 (1998). 


\section{TABLES}

TABLE I. $\alpha$-particle transition energies for various actinide isotopes. Ground state to ground state transition energies were taken from Audi et al. [21]. Calculated values for transitions from the second minima of the potential in the parent nuclei to the ground states and to the second and third minima in the daughters, and from the third minima in the parents to the third and second minima in the daughters, were taken from the predictions of Howard and Möller [12]. Numbers in bold are consistent with the experimental results (see text).

\begin{tabular}{|c|c|c|c|c|c|c|}
\hline $\begin{array}{l}\text { Mother } \\
\text { Isotope }\end{array}$ & $\begin{array}{l}\mathrm{E}_{\alpha} \\
\text { g.s. } \rightarrow \text { g.s. }\end{array}$ & $\begin{array}{l}\mathrm{E}_{\alpha} \\
\mathrm{II}^{\text {min }} \rightarrow \mathrm{II}^{\text {min }}\end{array}$ & $\begin{array}{l}\mathrm{E}_{\alpha} \\
\mathrm{II}^{\text {min }} \rightarrow \text { g.s. }\end{array}$ & $\begin{array}{l}\mathrm{E}_{\alpha} \\
\mathrm{II}^{\text {min }} \rightarrow \mathrm{III}^{\text {min }}\end{array}$ & $\begin{array}{l}\mathrm{E}_{\alpha} \\
\mathrm{III}^{\text {min }} \rightarrow \mathrm{III}^{\text {min }}\end{array}$ & $\begin{array}{l}\mathrm{E}_{\alpha} \\
\mathrm{III}^{\text {min }} \rightarrow \mathrm{II}^{\text {min }}\end{array}$ \\
\hline${ }^{238} \mathrm{Cm}$ & 6.51 & 5.90 & 8.25 & 4.38 & 5.24 & 6.76 \\
\hline${ }^{239} \mathrm{Cm}$ & 6.47 & 5.61 & 7.93 & 3.71 & 4.93 & 6.72 \\
\hline${ }^{240} \mathrm{Cm}$ & 6.29 & 5.68 & 7.80 & 3.65 & 4.57 & 6.61 \\
\hline${ }^{241} \mathrm{Cm}$ & 6.08 & 5.94 & 7.71 & 3.59 & 4.33 & 6.67 \\
\hline${ }^{237} \mathrm{Am}$ & 6.08 & 5.50 & 8.21 & 3.58 & 4.90 & 6.88 \\
\hline${ }^{238} \mathrm{Am}$ & 5.94 & 5.13 & 7.76 & 2.88 & 4.53 & 6.78 \\
\hline${ }^{239} \mathrm{Am}$ & 5.82 & 5.24 & 7.67 & 2.78 & 4.16 & 6.72 \\
\hline${ }^{240} \mathrm{Am}$ & 5.61 & 5.53 & 7.55 & 2.70 & 3.96 & 6.23 \\
\hline${ }^{241} \mathrm{Es}$ & 8.18 & 7.45 & 8.89 & 7.20 & 6.39 & 6.65 \\
\hline${ }^{242} \mathrm{Es}$ & 8.09 & 7.31 & 8.79 & 6.98 & 6.20 & 6.52 \\
\hline${ }^{243} \mathrm{Es}$ & 7.94 & 7.27 & 8.82 & 7.03 & 6.02 & 6.26 \\
\hline${ }^{244} \mathrm{Es}$ & 7.90 & 7.61 & 9.00 & 7.22 & 5.96 & 6.35 \\
\hline${ }^{245} \mathrm{Es}$ & 7.78 & 7.72 & 9.20 & 7.53 & 5.81 & 6.01 \\
\hline${ }^{246} \mathrm{Es}$ & 7.61 & 7.83 & 9.44 & 7.81 & 5.62 & 5.64 \\
\hline${ }^{247} \mathrm{Es}$ & 7.37 & 7.47 & 9.41 & 7.91 & 5.27 & 4.83 \\
\hline${ }^{242} \mathrm{Fm}$ & $8.31^{a}$ & 7.53 & 8.39 & 7.67 & 6.42 & 6.22 \\
\hline${ }^{243} \mathrm{Fm}$ & 8.55 & 7.74 & 8.66 & 7.85 & 6.53 & 6.48 \\
\hline${ }^{244} \mathrm{Fm}$ & 8.41 & 7.71 & 8.73 & 8.01 & 6.43 & 6.19 \\
\hline${ }^{245} \mathrm{Fm}$ & 8.30 & 7.95 & 8.86 & 8.08 & 6.28 & 6.15 \\
\hline${ }^{246} \mathrm{Fm}$ & 8.24 & 8.10 & 9.12 & 8.42 & 6.13 & 5.80 \\
\hline${ }^{247} \mathrm{Fm}$ & 8.06 & 8.18 & 9.39 & 8.73 & 5.90 & 5.35 \\
\hline${ }^{248} \mathrm{Fm}$ & 7.87 & 7.85 & 9.39 & 8.47 & 5.62 & 4.60 \\
\hline
\end{tabular}


TABLE II. Predicted half-lives for the 5.14, 5.27 and 5.53 MeV $\alpha$-particle groups seen in the Bk, Es and No-Lr sources respectively, assuming transitions from nuclei which are suggested in Table 1. Calculated values for transitions from the second minima of the potential in the parent nuclei to the second minima in the daughters, and from the third minima in the parents to the third minima in the daughters, are given ([9] and section III of the text). The observed half-lives are also compared to those calculated by the formulas of Viola and Seaborg [7].

\begin{tabular}{|c|c|c|c|c|c|}
\hline Transition & $\beta_{2}$ & $\beta_{3}$ & $\beta_{4}$ & $\mathrm{t}_{1 / 2}^{c a l}(\mathrm{~s})^{a}$ & $\mathrm{t}_{1 / 2}^{c a l} / \mathrm{t}_{1 / 2}^{e x p}$ \\
\hline \multicolumn{6}{|c|}{$\mathrm{E}_{\alpha}=5.14 \mathrm{MeV} ; \mathrm{t}_{1 / 2}^{\mathrm{exp}}=3.8 \pm 1 \mathrm{y}=1.2 \times 10^{8} \mathrm{~s}$} \\
\hline \multicolumn{6}{|c|}{${ }^{238} \mathrm{Am} \rightarrow{ }^{234} \mathrm{~Np} ; \mathrm{t}_{1 / 2}$ (V.S. $)^{b}=1.8 \times 10^{13} \mathrm{~s} ; \mathrm{t}_{1 / 2}^{\text {V.S. }} / \mathrm{t}_{1 / 2}^{e x p}=1.5 \times 10^{5}$} \\
\hline$\overline{\mathrm{II}^{\min } \rightarrow \mathrm{II}^{\min }}$ & $0.71^{c}$ & 0.0 & $0.09^{c}$ & $3.4 \times 10^{8}$ & 2.8 \\
\hline $\mathrm{III}^{\min } \rightarrow \mathrm{III}^{\min }$ & $1.05^{c}$ & $0.0^{d}$ & 0.0 & $1.0 \times 10^{8}$ & $8.3 \times 10^{-1}$ \\
\hline $\mathrm{III}^{\min } \rightarrow \mathrm{III}^{\min }$ & $1.05^{c}$ & $0.18^{e}$ & 0.0 & $4.5 \times 10^{6}$ & $3.8 \times 10^{-2}$ \\
\hline $\mathrm{III}^{\min } \rightarrow \mathrm{III}^{\min }$ & $0.85^{f}$ & $0.35^{f}$ & $0.18^{f}$ & $5.1 \times 10^{4}$ & $4.3 \times 10^{-4}$ \\
\hline \multicolumn{6}{|c|}{$\overline{\mathrm{E}_{\alpha}=5.14 \mathrm{MeV} ; \mathrm{t}_{1 / 2}^{\exp }=3.8 \pm 1 \mathrm{y}=1.2 \times 10^{8} \mathrm{~s}}$} \\
\hline \multicolumn{6}{|c|}{${ }^{238} \mathrm{Cm} \rightarrow{ }^{234} \mathrm{Pu} ; \mathrm{t}_{1 / 2}$ (V.S.) $)^{b}=4.5 \times 10^{12} \mathrm{~s} ; \mathrm{t}_{1 / 2}^{V . S .} / \mathrm{t}_{1 / 2}^{e x p}=3.8 \times 10^{4}$} \\
\hline $\mathrm{III}^{\text {min }} \rightarrow \mathrm{III}^{\min }$ & $1.05^{c}$ & $0.0^{d}$ & 0.0 & $3.3 \times 10^{8}$ & 2.7 \\
\hline $\mathrm{III}^{\text {min }} \rightarrow \mathrm{III}^{\text {min }}$ & $1.05^{c}$ & $0.17^{e}$ & 0.0 & $1.7 \times 10^{7}$ & $1.4 \times 10^{-1}$ \\
\hline $\mathrm{III}^{\min } \rightarrow \mathrm{III}^{\min }$ & $0.85^{f}$ & $0.35^{f}$ & $0.18^{f}$ & $1.5 \times 10^{5}$ & $1.2 \times 10^{-3}$ \\
\hline \multicolumn{6}{|c|}{ 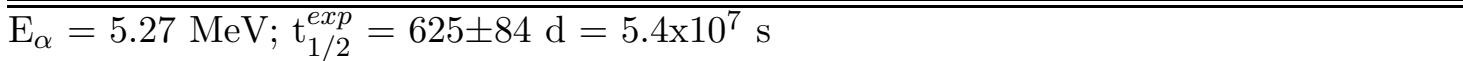 } \\
\hline \multicolumn{6}{|c|}{${ }^{247} \mathrm{Es} \rightarrow{ }^{243} \mathrm{Bk} ; \mathrm{t}_{1 / 2}$ (V.S. $)^{b}=1.5 \times 10^{14} \mathrm{~s} ; \mathrm{t}_{1 / 2}^{V . S .} / \mathrm{t}_{1 / 2}^{\exp }=2.8 \times 10^{6}$} \\
\hline$\overline{\mathrm{II}^{\min } \rightarrow \mathrm{II}^{\min }}$ & $0.71^{c}$ & 0.0 & $0.09^{c}$ & $3.9 \times 10^{9}$ & $7.2 \times 10^{1}$ \\
\hline $\mathrm{III}^{\min } \rightarrow \mathrm{III}^{\min }$ & $1.05^{c}$ & $0.0^{d}$ & 0.0 & $1.1 \times 10^{9}$ & $2.0 \times 10^{1}$ \\
\hline $\mathrm{III}^{\text {min }} \rightarrow \mathrm{III}^{\text {min }}$ & $1.05^{c}$ & $0.19^{e}$ & 0.0 & $3.3 \times 10^{7}$ & $6.1 \times 10^{-1}$ \\
\hline $\mathrm{III}^{\text {min }} \rightarrow \mathrm{III}^{\text {min }}$ & $0.85^{f}$ & $0.35^{f}$ & $0.18^{f}$ & $4.6 \times 10^{5}$ & $8.5 \times 10^{-3}$ \\
\hline \multirow{2}{*}{\multicolumn{6}{|c|}{$\begin{array}{l}\mathrm{E}_{\alpha}=5.53 \mathrm{MeV} ; \mathrm{t}_{1 / 2}^{\exp }=26 \pm 7 \mathrm{~d}=2.2 \times 10^{6} \mathrm{~s} \\
\left.{ }^{252} \mathrm{No} \rightarrow{ }^{248} \mathrm{Fm} ; \mathrm{t}_{1 / 2} \text { (V.S. }\right)^{b}=1.9 \times 10^{13} \mathrm{~s} ; \mathrm{t}_{1 / 2}^{V . S .} / \mathrm{t}_{1 / 2}^{e x p}=8.5 \times 10^{6}\end{array}$}} \\
\hline & & & & & \\
\hline$\overline{\mathrm{II}^{\min } \rightarrow \mathrm{II}^{\min }}$ & $0.79^{c}$ & 0.0 & $0.14^{c}$ & $3.6 \times 10^{8}$ & $1.6 \times 10^{2}$ \\
\hline $\mathrm{III}^{\text {min }} \rightarrow \mathrm{III}^{\text {min }}$ & $1.2^{c}$ & $0.0^{d}$ & 0.0 & $1.9 \times 10^{8}$ & $8.5 \times 10^{1}$ \\
\hline $\mathrm{III}^{\min } \rightarrow \mathrm{III}^{\min }$ & $1.2^{c}$ & $0.19^{e}$ & 0.0 & $7.0 \times 10^{6}$ & 3.1 \\
\hline $\mathrm{III}^{\min } \rightarrow \mathrm{III}^{\min }$ & $0.85^{f}$ & $0.35^{f}$ & $0.18^{f}$ & $2.3 \times 10^{5}$ & $1.0 \times 10^{-1}$ \\
\hline
\end{tabular}

${ }^{a}$ See text.

${ }^{b}$ Ref. [7].

${ }^{c} \epsilon_{2}$ and $\epsilon_{4}$ were taken from Ref. [12] and converted to $\beta_{2}$ and $\beta_{4}$ according to Ref. [24].

$d$ Assuming $\beta_{3}=0$.

e Assuming $\beta_{3}=\epsilon_{3}$ of Ref. [12].

$f$ Parameter given by Ćwiok et al. [25] for the hyperdeformed state of ${ }^{232} \mathrm{Th}$. 
TABLE III. Half-lives of some isomeric states and their ratios to the half-lives of their corresponding normal-deformed ground states.

\begin{tabular}{llll}
\hline \hline Isotope & $\mathrm{t}_{1 / 2}$ (g.s.) & $\mathrm{t}_{1 / 2}($ i.s. $)$ & $\mathrm{t}_{1 / 2}(\mathrm{i} . \mathrm{s}.) / \mathrm{t}_{1 / 2}$ (g.s.) \\
\hline${ }^{236} \mathrm{Bk}$ & $42.4 \mathrm{~s}^{a}$ & $\geq 30 \mathrm{~d}^{b}$ & $\geq 6.1 \times 10^{4}$ \\
${ }^{236} \mathrm{Am}$ & $3.7 \mathrm{~m}(?)^{c}$ & $219 \mathrm{~d}^{b}$ & $8.5 \times 10^{4}$ \\
${ }^{238} \mathrm{Am}^{d}$ & $98 \mathrm{~m}^{g}$ & $3.8 \mathrm{y}$ & $2.0 \times 10^{4}$ \\
${ }^{247} \mathrm{Es}^{e}$ & $4.55 \mathrm{~m}^{g}$ & $625 \mathrm{~d}$ & $2.0 \times 10^{5}$ \\
${ }^{252} \mathrm{No}^{f}$ & $2.3 \mathrm{~s}^{g}$ & $26 \mathrm{~d}$ & $9.8 \times 10^{5}$ \\
\hline \hline
\end{tabular}

${ }^{a}$ Predicted by P. Möller, J. R. Nix and K.-L. Kratz, At. Data Nucl. Data Tables 66, 131 (1997).

${ }^{b}$ Ref. [2].

${ }^{c}$ G. Pfennig, H. Klewe-Nebenius and W. Seelmann-Eggebert, Karlsruher Nuklidkarte (1995).

${ }^{d}$ Assuming that the $5.14 \mathrm{MeV}$ is from ${ }^{238} \mathrm{Am}$ (see table 1 ).

${ }^{e}$ Assuming that the $5.27 \mathrm{MeV}$ is from ${ }^{247} \mathrm{Es}$ (see table 1).

$f$ Assuming that the $5.53 \mathrm{MeV}$ is from ${ }^{252} \mathrm{No}$ (see text).

$g$ R. B. Firestone et al., Table of Isotopes, Wiley-Interscience (1996). 
TABLE IV. Estimated production cross sections of various actinide nuclei in their isomeric states via secondary reactions. Estimation for the superheavy isotope ${ }^{272} 112$ is also given.

\begin{tabular}{|c|c|c|c|c|c|c|c|}
\hline Isotope & $\begin{array}{l}\text { No. of } \\
\text { Atoms }\end{array}$ & $\begin{array}{l}\sigma_{T} \\
\text { (barn) }\end{array}$ & Reactions & $\begin{array}{l}\mathrm{E}_{x}(\mathrm{C} . \mathrm{N} .)^{a} \\
(\mathrm{MeV})\end{array}$ & $\begin{array}{l}\sigma_{\text {frag. }}{ }^{b} \\
(\mathrm{mb})\end{array}$ & $\mathrm{f}^{c}$ & $\begin{array}{l}\sigma_{R}(\text { H.I. })^{d} \\
(\mathrm{mb})\end{array}$ \\
\hline$\overline{236} \mathrm{Am}$ & $3.1 \times 10^{5}$ & $8.2 \times 10^{-13}$ & $\begin{array}{l}{ }^{182-184,186} \mathrm{~W}\left({ }^{55-53,51} \mathrm{Ti}, \mathrm{p}\right) \\
{ }^{182-184,186} \mathrm{~W}\left({ }^{56-54,52} \mathrm{Ti}, \mathrm{pn}\right) \\
{ }^{182-184,186} \mathrm{~W}\left({ }^{58-56,54} \mathrm{~V}, \alpha\right) \\
{ }^{182-184,186} \mathrm{~W}\left({ }^{59-57,55} \mathrm{~V}, \alpha \mathrm{n}\right)\end{array}$ & $29.8-38.0$ & $0.77-0.01$ & $1 \times 10^{-2}$ & 12.8 \\
\hline${ }^{236} \mathrm{Bk}$ & $4.4 \times 10^{4}$ & $1.2 \times 10^{-13}$ & $\begin{array}{l}{ }^{182-184,186} \mathrm{~W}\left({ }^{55-53,51} \mathrm{Cr}, \mathrm{p}\right) \\
{ }^{182-184,186} \mathrm{~W}\left({ }^{56-54,52} \mathrm{Cr}, \mathrm{pn}\right) \\
{ }^{182-184,186} \mathrm{~W}\left({ }^{58-56,54} \mathrm{Mn}, \alpha\right) \\
{ }^{182-184,186} \mathrm{~W}\left({ }^{59-57,55} \mathrm{Mn}, \alpha \mathrm{n}\right)\end{array}$ & $30.9-42.2$ & $5.5-0.4$ & $5 \times 10^{-3}$ & 0.22 \\
\hline${ }^{238} \mathrm{Bk}$ & $3.3 \times 10^{4}$ & $8.9 \times 10^{-14}$ & $\begin{array}{l}{ }^{182-184,186} \mathrm{~W}\left({ }^{57-55,53} \mathrm{Cr}, \mathrm{p}\right) \\
{ }^{182-184,186} \mathrm{~W}\left({ }^{58-56,54} \mathrm{Cr}, \mathrm{pn}\right) \\
{ }^{182-184,186} \mathrm{~W}\left({ }^{60-58,56} \mathrm{Mn}, \alpha\right) \\
182-184,186 \mathrm{~W}\left({ }^{61-59,57} \mathrm{Mn}, \alpha \mathrm{n}\right)\end{array}$ & $33.1-36.9$ & $4.0-0.09$ & $5 \times 10^{-3}$ & 0.26 \\
\hline${ }^{247} \mathrm{Es}$ & $1.4 \times 10^{4}$ & $3.7 \times 10^{-14}$ & $\begin{array}{l}{ }^{182-184,186} \mathrm{~W}\left({ }^{66-64,62} \mathrm{Fe}, \mathrm{p}\right) \\
182-184,186 \mathrm{~W}\left({ }^{67-65,63} \mathrm{Fe}, \mathrm{pn}\right) \\
{ }^{182-184,186} \mathrm{~W}\left({ }^{69-67,65} \mathrm{Co}, \alpha\right) \\
182-184,186 \mathrm{~W}\left({ }^{70-68,66} \mathrm{Co}, \alpha \mathrm{n}\right)\end{array}$ & $33.3-31.4$ & $0.24-0.006$ & $2.6 \times 10^{-3}$ & 6.2 \\
\hline${ }^{252} \mathrm{No}$ & $1.4 \times 10^{4}$ & $3.7 \times 10^{-14}$ & $\begin{array}{l}{ }^{182-184,186} \mathrm{~W}\left({ }^{71-69,67} \mathrm{Cu}, \mathrm{p}\right) \\
{ }^{182-184,186} \mathrm{~W}\left({ }^{72-70,68} \mathrm{Cu}, \mathrm{pn}\right) \\
{ }^{182-184,186} \mathrm{~W}\left({ }^{74-72,70} \mathrm{Zn}, \alpha\right) \\
{ }^{182-184,186} \mathrm{~W}\left({ }^{75-73,71} \mathrm{Zn}, \alpha \mathrm{n}\right)\end{array}$ & $24.4-27.4$ & $0.88-0.03$ & $1 \times 10^{-3}$ & 4.0 \\
\hline$\overline{272} 112$ & $\sim 5 \times 10^{2 e}$ & $1.3 \times 10^{-15}$ & ${ }^{182-184,186} \mathrm{~W}\left({ }^{90-88,86} \mathrm{Sr}, \gamma\right)$ & $2.0-9.8^{f}$ & $1.8-0.05^{g}$ & $5 \times 10^{-5}$ & 3.8 \\
\hline
\end{tabular}

a Excitation energies of the compound nuclei produced by the ${ }^{182} \mathrm{~W}(\mathrm{X}, \mathrm{p})$ (left) and the ${ }^{186} \mathrm{~W}(\mathrm{X}, \mathrm{p})$ (right) reactions are given, assuming bombarding energies which are equal to the Coulomb barriers between the corresponding projectile and target nuclei $\left(\mathrm{r}_{0}=1.4 \mathrm{fm}\right)$.

${ }^{b}$ Fragment cross sections were estimated according to the data of Ref. [26] (see text).

$c$ The value of " $\mathrm{f}$ ", the ratio of the production cross section for a high energy fragment $\left(\mathrm{E}_{\text {Lab }} \gtrsim 4.2 \mathrm{MeV} /\right.$ nucleon) to the total production cross section of the particular fragment, was assumed to be $1 \%$ for the fragments which produced the ${ }^{236} \mathrm{Am}$ isotope, and reduced exponentially to $0.1 \%$ for those which produced the ${ }^{252}$ No isotope (see text).

${ }^{d}$ The same cross section was assumed for the various heavy ion reactions which contribute to the production of a particular isotope.

e Ref. [5].

$f$ As "a" above but for the ${ }^{182} \mathrm{~W}\left({ }^{90} \mathrm{Sr}, \gamma\right)$ (left) and ${ }^{186} \mathrm{~W}\left({ }^{86} \mathrm{Sr}, \gamma\right)$ (right) reactions. The mass of the ${ }^{272} 112$ nucleus was taken from S. Liran and N. Zeldes, At. Data Nucl. Data Tables 17, 431 (1976).

$g$ Taken from U. Trabitzsch and K. Bächmann, Radiochim. Acta 16, 15 (1971). 


\section{FIGURES}

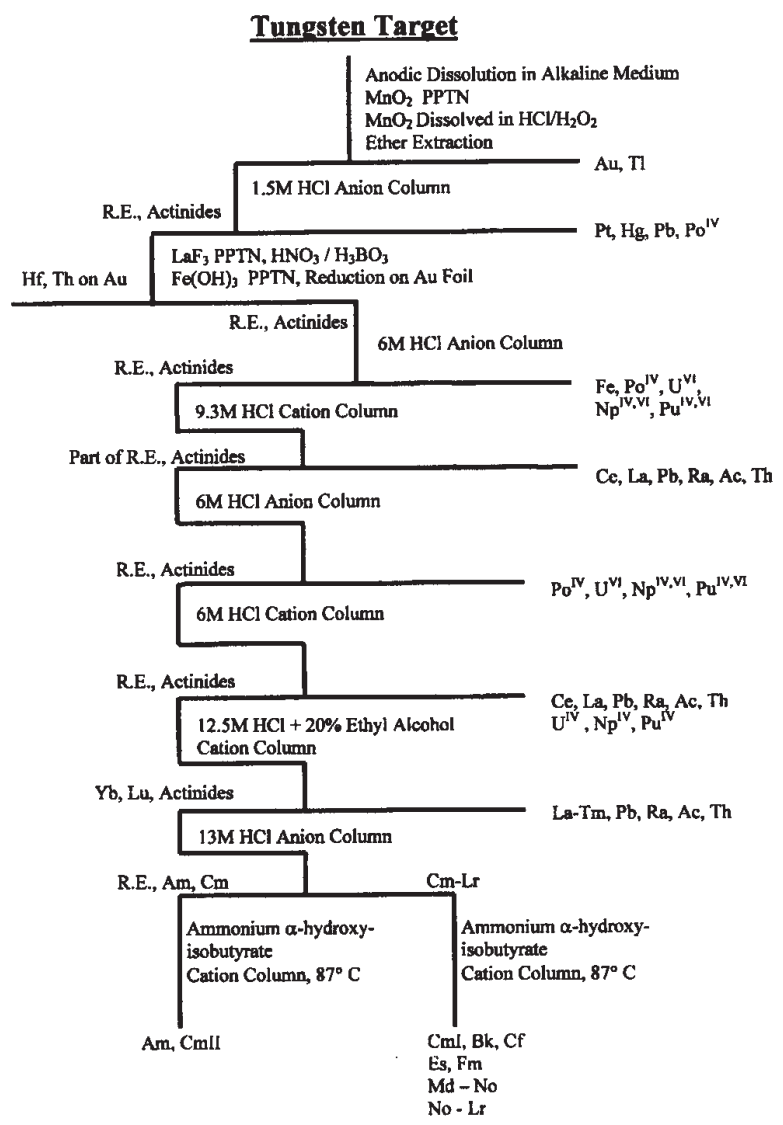

FIG. 1. Block diagram of the chemical separation of the actinide fraction from the $\mathrm{W}$ target. 


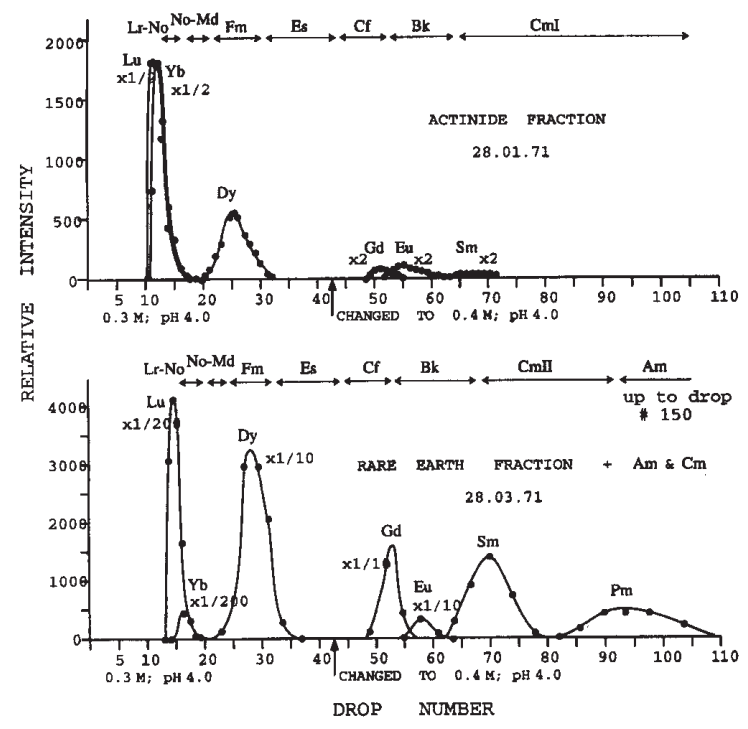

FIG. 2. Relative elution positions of rare earth elements from Dowex-50 with $\mathrm{pH} 4.0$ ammonium $\alpha$-hydroxyisobutyrate at $87^{\circ} \mathrm{C}$. Top: Actinide fraction. Bottom: Rare earth fraction + Am and $\mathrm{Cm}$.
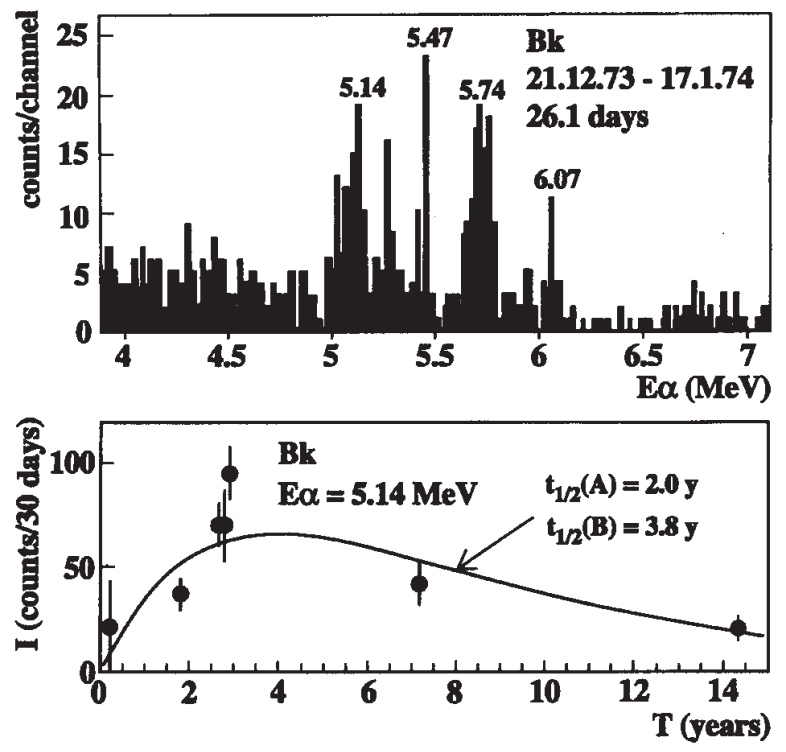

FIG. 3. Top: $\alpha$-particle spectrum obtained with the Bk source. Bottom: Decay curve of the 5.14-MeV group shown in the top figure. 

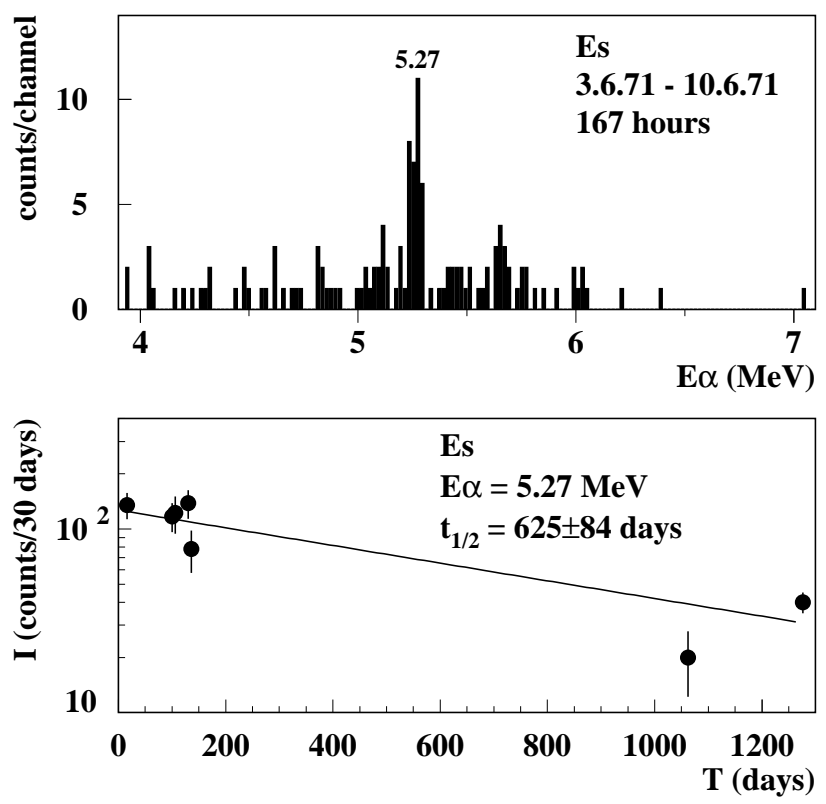

FIG. 4. Top: $\alpha$-particle spectrum obtained with the Es source. Bottom: Decay curve of the $5.27-\mathrm{MeV}$ group shown in the top figure.

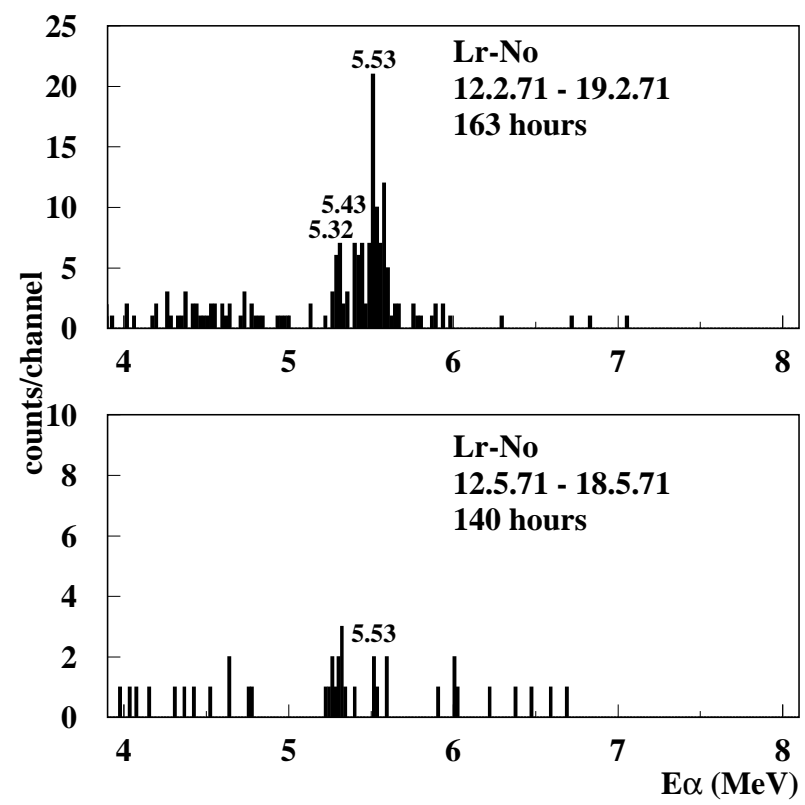

FIG. 5. Top: $\alpha$-particle spectrum obtained with the Lr-No source. Bottom: The same as above but taken about 3 months later. 

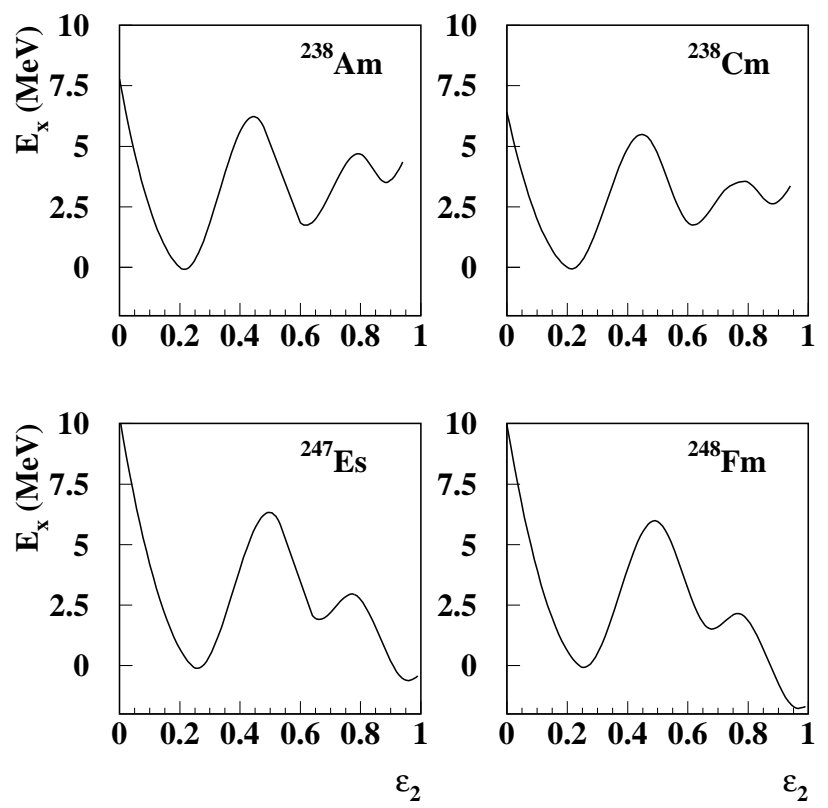

FIG. 6. Potential energies as function of quadrupole deformations for 4 nuclei according to ref. $[12]$.

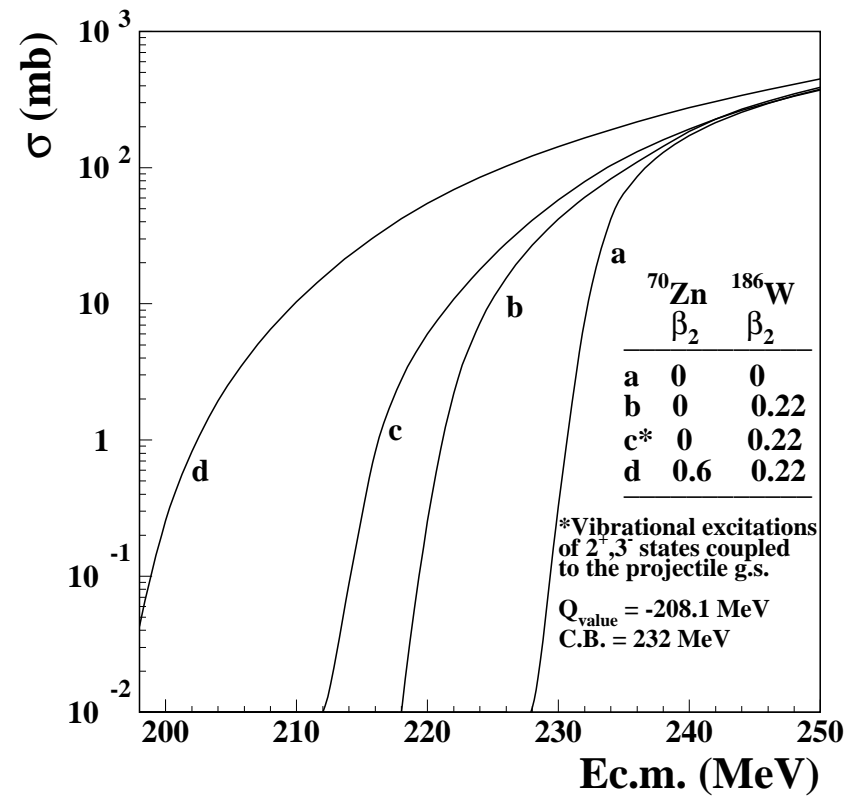

FIG. 7. Calculated fusion cross sections using the Code CCDEF [28] for the ${ }^{70} \mathrm{Zn}+{ }^{186} \mathrm{~W}$ reaction assuming various quadrupole deformations of the projectile and target nuclei (see text). 


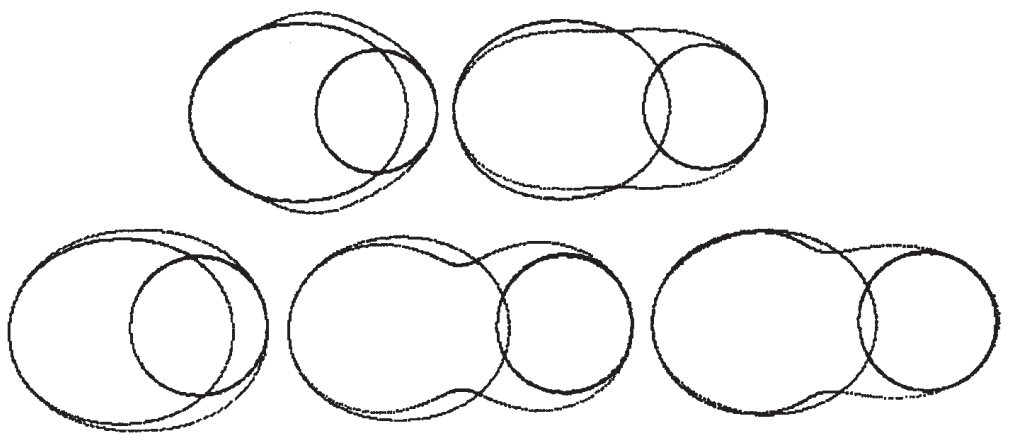

FIG. 8. Calculated shapes of two compound nuclei at various configurations together with the shapes of the corresponding projectile and target nuclei. Top, left: $\mathrm{A}_{C . N} .=239$ in the normal ground state; $\beta_{2}=0.2 ; \beta_{4}=0.08$ [12]. Top, right: $\mathrm{A}_{C . N} .=239$ in the second minimum; $\beta_{2}=$ $0.77 ; \beta_{4}=0.1$ [12]. In both figures: $\mathrm{A}_{\text {heavy }}=186 ; \beta_{2}=0.22$ [29]. $\mathrm{A}_{\text {light }}=53 ; \beta_{2}, \beta_{3}, \beta_{4}=0.0$. Bottom, left: $\mathrm{A}_{C . N}$. $=253$ in the normal ground state; $\beta_{2}=0.28 ; \beta_{4}=0.01$ [12]. Bottom, center: $\mathrm{A}_{C . N .}=253$ in the third minimum; $\beta_{2}=1.2 ; \beta_{4}=0.0[12]$. Bottom, right: $\mathrm{A}_{C . N} .=253$ with parameters of the third minimum of ${ }^{232} \mathrm{Th} ; \beta_{2}=0.85 ; \beta_{3}=0.35 ; \beta_{4}=0.18$ [25]. In the three figures at the bottom: $\mathrm{A}_{\text {heavy }}=186 ; \beta_{2}=0.22[29] . \mathrm{A}_{\text {light }}=67 ; \beta_{2}, \beta_{3}, \beta_{4}=0.0$. 